\title{
Systematic effects in measurement of black hole masses by emission-line reverberation of active galactic nuclei: Eddington ratio and inclination
}

\author{
S. Collin ${ }^{1}$, T. Kawaguchi ${ }^{2}$, B. M. Peterson ${ }^{3}$, and M. Vestergaard ${ }^{4}$ \\ 1 LUTH, Observatoire de Paris, Section de Meudon, 92195 Meudon Cedex, France \\ e-mail: suzy.collin@obspm.fr \\ 2 Optical and Infrared Division, NAOJ, Mitaka, Tokyo 181-8588, Japan \\ 3 Department of Astronomy, The Ohio State University, 140 West 18th Avenue, Columbus, OH 43210, USA \\ 4 Steward Observatory, University of Arizona, 933 N. Cherry Avenue, Tucson, AZ 85721, USA
}

Received 18 January 2006 / Accepted 24 March 2006

ABSTRACT

\begin{abstract}
Context. Scatter around the relationship between central black hole masses in active galactic nuclei (AGNs) obtained by reverberationmapping methods and host-galaxy bulge velocity dispersion indicates that the masses are uncertain typically by a factor of about three. Aims. In this paper, we try to identify the sources and systematics of this uncertainty.

Methods. We characterize the broad $\mathrm{H} \beta$ emission-line profiles by the ratio of their full-width at half maximum $(F W H M)$ to their line dispersion, i.e., the second moment of the line profile. We use this parameter to separate the reverberation-mapped AGNs into two populations, the first with narrower $\mathrm{H} \beta$ lines that tend to have relatively extended wings, and the second with broader lines that are relatively flat-topped. The first population is characterized by higher Eddington ratios than the second. Within each population, we calibrate the black-hole mass scale by comparison of the reverberation-based mass with that predicted by the bulge velocity dispersion. We also use the distribution of ratios of the reverberation-based mass to the velocity-dispersion mass prediction in a comparison with a "generalized thick disk" model in order to see if inclination can plausibly account for the observed distribution.

Results. We find that the line dispersion is a less biased parameter in general than $F W H M$ for black hole mass estimation, although we show that it is possible to empirically correct for the bias introduced by using $F W H M$ to characterize the emission-line width. We also argue that inclination effects are apparent only in some small subset of the reverberation-based mass measurements; it is primarily the objects with the narrowest emission lines that seem to be most strongly affected.

Conclusions. Our principal conclusion is that the $\mathrm{H} \beta$ profile is sensitive primarily to Eddington ratio, but that inclination effects play a role in some cases.
\end{abstract}

Key words. galaxies: quasars: general - galaxies: nuclei - galaxies: Seyfert

\section{Introduction}

During the last twenty years, reverberation mapping (Blandford \& McKee 1982; Peterson 1993) of the broad emission lines in active galactic nuclei (AGNs) has been used to determine the size of the broad-line region (BLR) in these objects. By combining the BLR size with the emission-line Doppler width, it is possible to estimate the mass of the central source, presumed to be a black hole $(\mathrm{BH})$, as

$M_{\mathrm{BH}}=f \frac{R_{\mathrm{BLR}} \Delta V^{2}}{G}$,

where $R_{\mathrm{BLR}}$ is the size of the BLR, $\Delta V$ is the emission line width, and $f$ is a scale factor of order unity that depends on the structure, kinematics, and inclination of the BLR. In the cases where multiple emission lines have been observed, it is found that the higher-ionization lines tend to have shorter response times, or lags $\tau$, and thus arise in regions relatively closer to the central source than the lower-ionization lines. Moreover, the lines with shorter lags tend to be broader than those with longer lags, generally consistent with the virial prediction $\tau=$ $R / c \propto \Delta V^{-2}$, providing a strong argument that Eq. (1) is valid (Peterson \& Wandel 1999, 2000; Onken \& Peterson 2002;
Kollatschny 2003a). In addition, AGNs show a relationship between $\mathrm{BH}$ mass and the host-galaxy bulge velocity dispersion $\sigma_{*}$ (Gebhardt et al. 2000b; Ferrarese et al. 2001; Onken et al. 2004; Nelson et al. 2004) that is consistent with this same correlation, the $M_{\mathrm{BH}}-\sigma_{*}$ relationship, that is observed in quiescent galaxies (Ferrarese \& Merritt 2000; Gebhardt et al. 2000a; Tremaine et al. 2002).

Reverberation mapping has also confirmed the expected relationship between the size of the BLR and the luminosity of the AGN, which takes the form

$R_{\mathrm{BLR}} \propto L^{\alpha}$,

where $\alpha \approx 0.5$, but depends somewhat on which luminosity measure is being used and possibly also on which emission line is used to determine $R_{\mathrm{BLR}}$ (Wandel et al. 1999; Kaspi et al. 2000, 2005; Bentz et al. 2006). This radius-luminosity $(R-L)$ relationship is important not only because it tells us something about the physics of the emission-line region, but because it also affords an almost trivially simple secondary mass indicator, since through Eq. (2), measurement of the luminosity provides a surrogate for the otherwise hard-to-measure BLR radius. It thus becomes possible to easily estimate BH masses for large samples of AGNs (Wandel et al. 1999; Vestergaard 2002, 2004; 
McLure \& Jarvis 2002; Kollmeier et al. 2006; Vestergaard \& Peterson 2006). Such studies have shown, for example, that the $\mathrm{BH}$ masses of high-redshift quasars are very large (Vestergaard 2004; Netzer 2003). At the other extreme, very small BH masses have been inferred for low-luminosity Seyferts (Greene \& Ho 2004; Barth et al. 2005). It has also been found that application of luminosity-scaling relationships indicate that AGNs with small line widths, i.e., narrow-line Seyfert 1 (NLS1) galaxies have relatively small $\mathrm{BH}$ masses for their luminosity. It appears that the ratio of their bolometric luminosity to the Eddington luminosity, which we hereafter refer to as the "Eddington ratio," is high, close in fact to unity. Through accretion-disk modeling, Collin et al. (2002), Kawaguchi (2003) and Collin \& Kawaguchi (2004) have shown that NLS1s have accretion rates larger than the Eddington value (although also see Williams et al. 2004).

These results have important physical and cosmological consequences (cf. Yu \& Tremaine 2003; Kawaguchi et al. 2004b). It is therefore essential to understand the accuracy of the reverberation measurements (primary mass indicators) and their calibration uncertainties as well as the scaling relationships (secondary mass indicators) based on them, especially when these are extrapolated beyond the range over which they were determined, i.e., to much higher and much lower luminosities, to high redshifts, and to high and low Eddington ratios. In principle, this could be achieved through comparison with an independent primary or secondary mass indicator, though at the present time there is no other obvious choice beyond the $M_{\mathrm{BH}}-\sigma_{*}$ relationship. Indeed, the $M_{\mathrm{BH}}-\sigma_{*}$ relationship has been employed in investigations of AGN BH masses in two distinct ways, (a) by using measurements of $\sigma_{*}$ to infer a $\mathrm{BH}$ mass to compare with the reverberation-based mass in an attempt to extract information on the inclination of the BLR (Wu \& Han 2001; Zhang \& Wu 2002), and (b) by normalizing the AGN $M_{\mathrm{BH}}-\sigma_{*}$ relationship to that of quiescent galaxies in order to calibrate the reverberationbased mass scale by determining a statistical average value for the scaling factor $\langle f\rangle$ (Onken et al. 2004).

Of course, it would be even more desirable to use reverberation-mapping techniques to obtain a velocity-delay map that would reveal the kinematics and structure of the BLR and lead to determination of the central mass. However, various limitations of the reverberation data obtained to date have precluded this (Horne et al. 2004), but have nevertheless yielded mean response times for emission lines and have enabled BH mass estimates through Eq. (1). This apparent simplication entails a price in the accuracy to which the BH mass can be measured and a number of ambiguities arise. Among the more challenging are:

1. What line-width measure gives the most accurate BH mass? The two commonly used measures are the full width at half maximum (FWHM) and the second moment of the emissionline profile $\sigma_{\text {line }}$, which we refer to as the "line dispersion" ${ }^{1}$." The ratio of these two measures varies widely from one AGN to another and, as discussed below, varies with other AGN spectral properties. Thus, depending on which measure we select, we might be introducing a bias in mass estimate that varies with other AGN parameters.

2. How does one evaluate and interpret the scaling factor $f$ ? It is clear that $f$ should depend strongly on the inclination for a flattened system (e.g., a rotating disk), but it may vary with other AGN properties. Given that we are characterizing the size and velocity dispersion of the BLR by single numbers,

1 The line dispersion for an emission line $\sigma_{\text {line }}$ should not be confused with the host galaxy bulge velocity disperion $\sigma_{*}$. we are subsuming a lot of our ignorance of AGN structure into this single parameter, so interpretation can be difficult and ambiguous.

In this contribution, we will examine the systematics of the linewidth measures and attempt to identify possible biases associated with the character of the emission-line profiles. We will also, in the spirit of an exercise, assume that the $\mathrm{H} \beta$-emitting component of the BLR is an azimuthally symmetric, relatively flat structure that is dominated by rotational motion (i.e., a Keplerian disk). We will combine this with an isotropic component, a wind perhaps, and investigate the effects of disk inclination on the scaling factor $f$. Our goal is to attempt to disentangle the different factors which play a role in determining line profile of AGNs, and therefore in the scale factor and in the mass determination.

As we noted earlier, we subsume most of our ignorance about the BLR structure and kinematics into the scale factor $f$ and separate it from the "observable" quantity in Eq. (1), which we refer to as the "virial product," $V P=c \tau \Delta V^{2} / G$, which has units of mass and differs from the actual BH mass only by the dimensionless factor $f$. In this contribution, we will not concern ourselves with uncertainties in the virial product, which are generally $\sim 30 \%$. It is the much larger uncertainty in $f$ that will concern us here.

In the next section, we study the relationship between $F W H M$ and $\sigma_{\text {line }}$ and we show that the ratio of these quantities varies strongly among the objects. We try to understand the cause and the implications of these variations. By comparing the virial products with the $\mathrm{BH}$ masses determined through the $M_{\mathrm{BH}}-\sigma_{*}$ relationship, we consider the relationships among the scale factor, the line-width ratio $F W H M / \sigma_{\text {line }}$, and the Eddington ratio. In Sect. 3, we discuss the influence of the inclination of the BLR on the scale factor. Within the context of our simple two-component model, we will argue that some objects, specifically some of those for which $F W H M / \sigma_{\text {line }}$ is small, are likely seen nearly face-on geometry, and consequently their BH masses could be significantly underestimated. In Sect. 4, we speculate on the physical conditions in the BLR that could explain our results. We conclude with a summary in Sect. 5.

\section{Normalization of reverberation masses: the scale factor $f$}

The fundamental challenge that we need to address is how to obtain from reverberation data masses that are accurate and unbiased with respect to the various factors, such as inclination of the BLR, that can affect the value of the virial product. Given our poor understanding of the structure and kinematics of the BLR we do not know a priori what these various factors might be. We do know, however, that the broad $\mathrm{H} \beta$ profiles show marked differences among AGNs, and it is certainly true that the mass we adopt for a particular AGN will depend on precisely how we characterize the width of the line. Thus, our approach will be necessarily empirical: we will examine various ways of determining the line-width measure that is used as $\Delta V$ in Eq. (1) and attempt to identify systematic effects or biases.

\subsection{Mean and rms spectra}

In computing reverberation-based masses, it is common practice to take all of the individual spectra that were measured to obtain the continuum and emission-line light curves and construct "mean" and "root-mean-square (rms)" spectra. The advantage of 
the rms spectrum over the mean spectrum is that it isolates the spectral components that are actually varying, and automatically removes constant or slowly varying components, such as the narrow emission lines that arise on much larger physical scales. The disadvantage of using the rms spectrum is that it is often quite noisy as the amplitude of spectral variability is usually rather low. It is consequently not obvious whether it is better to measure the line width in the mean or the rms spectrum. We will argue here that use of the mean spectrum for line-width measurement gives results consistent with line-width measurements based on rms spectra, provided that one can account for contaminating features, the narrow-line components being most important in the case of the Balmer lines. This is important because in using single-epoch spectra and scaling relationships to estimate $\mathrm{BH}$ masses, the variable part of the emission line cannot be isolated.

\subsection{Line-width measures: FWHM and $\sigma_{\text {line }}$}

We first consider the differences between the $F W H M$ and the line dispersion $\sigma_{\text {line }}$. The $F W H M$ is a zeroth-order moment of the profile and the line dispersion is a second-order moment that is relatively more sensitive to the line wings and less sensitive to the line core. It is traditional in AGN studies to use FWHM to characterize line widths. For determination of $\mathrm{BH}$ masses, Wandel et al. (1999) and Kaspi et al. (2000) used FWHM, but based on a suggestion by Fromerth \& Melia (2000), Peterson et al. (2004) investigated use of the line dispersion and found that it can be measured to higher precision (i.e., with the smaller formal uncertainty) than $F W H M$, especially in noisy spectra, and that the virial relationship $\tau \propto \Delta V^{-2}$ is better reproduced with $\sigma_{\text {line }}$ than with $F W H M$. From the point of view of accuracy (i.e., a measurement closest to the true value), however, it is not clear which is the best line-width measure to use in Eq. (1), and it is that question we take up here.

The relationship between $\sigma_{\text {line }}$ and $F W H M$ depends on the line profile: it is well-known, for example, that for a Gaussian profile $F W H M / \sigma_{\text {line }}=2(2 \ln 2)^{1 / 2}=2.35$. For a rectangular function, $F W H M / \sigma_{\text {line }}=2 \sqrt{3}=3.46$, and a triangular function has $F W H M / \sigma_{\text {line }}=\sqrt{6}=2.45$. Similarly, for an edge-on rotating ring, $F W H M / \sigma_{\text {line }}=2 \sqrt{2}=2.83$. At the lower extreme, $F W H M / \sigma_{\text {line }} \rightarrow 0$ for a Lorentzian profile.

Peterson et al. (2004) provide measurements of FWHM and $\sigma_{\text {line }}$ from the rms spectra for all the lines for which time-delay measurements are available for virtually all of the reverberation-mapped AGNs (see their Table 6). For the purpose of comparison, we have measured the $\mathrm{H} \beta$ line widths in the corresponding mean spectra, using the same algorithms and assumptions of Peterson et al. (2004) and removing the narrowline components whenever necessary. Also, the $\mathrm{H} \beta$ profiles in the mean spectra often have strong contamination in the longwavelength wing, underneath the [O III] $\lambda \lambda 4959,5007$ lines, by Fe II m42 emission. This contamination is much weaker in the rms spectra because Fe II emission seems to be less variable than the Balmer lines (cf. Vestergaard \& Peterson 2005). For this reason, the $\sigma_{\text {line }}$ measurements we use are based on the short-wavelength side of the line, assuming that line is approximately symmetric. These measurements, plus the $\mathrm{H} \beta$ rms spectrum measurements from Peterson et al. (2004), are given in Table 1, and these values will be used throughout the rest of this paper. Columns (1) and (2) identify the AGN and time interval (Julian Date - 2400000) during which the data were obtained, respectively. Column (3) gives the cross-correlation function centroid $\tau_{\text {cent }}$, in days, which is our preferred measure of the emission-line lag. Column (4) gives an estimate of the uncertainty $\Delta \tau_{\text {cent }}$, that is the root mean square of the usually slightly asymmetric upward and downward uncertainties, again in days. Columns (5) give the logarithm of the mean optical luminosity in $\mathrm{erg} \mathrm{s}^{-1}$ based on the continuum measurements made during the interval given in Col. (2), and Col. (6) gives the rms variability of the luminosity during the same interval. The following columns give the values and uncertainties all in $\mathrm{km} \mathrm{s}^{-1}$ in the rest frame of the AGN and corrected for the spectrograph resolution, for $F W H M$ in the mean spectrum (Cols. 7 and 8$), \sigma_{\text {line }}$ in the mean spectrum (Cols. 9 and 10), FWHM in the rms spectrum (Cols. 11 and 12), and $\sigma_{\text {line }}$ in the rms spectrum (Cols. 13 and 14), We note that the optical luminosity has not been corrected for the host-galaxy starlight contribution. Therefore, the luminosity and Eddington ratios, especially for the less-luminous objects, are subject to overestimation on account of starlight contamination. Some of the values in this table will be superseded by work in progress, but since the database is constantly evolving, we have decided to proceed with the present data, as our conclusions are not likely to change drastically by a few more precise measurements.

In Fig. 1, we compare measurements of $\sigma_{\text {line }}$ for $\mathrm{H} \beta$ in the mean and rms spectra (left panel) and $F W H M$ for $\mathrm{H} \beta$ in the mean and rms spectra (right panel). Both $F W H M$ and $\sigma_{\text {line }}$ measurements in the rms spectra have much larger error bars since the rms spectra tend to be noisy. But we do see that for both line-width measures the widths of the $\mathrm{H} \beta$ line in the mean and rms spectra are well correlated, though the lines are typically $\sim 20 \%$ broader in the mean spectra. This is a well-documented phenomenon (e.g., Sergeev et al. 1999; Shapovalova et al. 2004). It has been suggested by Shields et al. (1995) that the highest velocity gas in the BLR is optically thin, and this could account for the lower level of variability in the line wings.

In Fig. 2, we compare the virial product based on $\sigma_{\text {line }}$, equal to $c \tau \sigma_{\text {line }}^{2} / G$ (hereafter $V P_{\mathrm{s}}$ ), versus the virial product based on $F W H M$, equal to $c \tau F W H M^{2} / G$ (hereafter $V P_{\mathrm{f}}$ ), for all the datasets in Table 1, except for four data sets for which the lag uncertainty is very large, with $\Delta \tau_{\text {cent }}>\tau_{\text {cent }}$, i.e., PG $0844+349$, NGC 3227, NGC 4593 and IC 4329A. While the virial products based on FWHM are well-correlated with those based on $\sigma_{\text {line }}$, there is scatter of about a factor of three in each case, reflecting the broad range of values of $F W H M / \sigma_{\text {line }}$ in these datasets (cf. Fig. 9 of Peterson et al. 2004).

The immediate question now becomes which one of the linewidth measures ought to be used to compute the BH mass? To investigate this further, we considered how the line-width ratio $F W H M / \sigma_{\text {line }}$ is correlated with other spectral properties. In Fig. 3, we show for $\mathrm{H} \beta F W H M / \sigma_{\text {line }}$ as a function of the line width $\sigma_{\text {line }}$ in both the mean and rms spectra. The results for the mean and the rms spectrum are quite similar. In both cases, the ratio has a large dispersion, but $F W H M / \sigma_{\text {line }}$ shows a clear tendency to be smaller than 2.35, the ratio for a Gaussian profile, for narrow-line objects, and larger than 2.35 for broad-line objects. Figure 3 tells us that the broad-line objects have more flat-topped profiles, while the narrow-line objects have more extended wings, relative to a Gaussian; in other words, this ratio correlates with the characteristics of the line profile, which in turn correlate with other spectral properties as is sometimes embodied in "Eigenvector 1" from principal component analysis (e.g., Boroson \& Green 1992), as is well known. We can somewhat arbitrarily separate the AGNs into two "populations" based on line-width ratio, Population 1 with $F W H M / \sigma_{\text {line }}<$ 2.35 and Population 2 with $F W H M / \sigma_{\text {line }}>2.35$. As seen in 
Table 1. Principal quantities of the reverberation mapped AGNs.

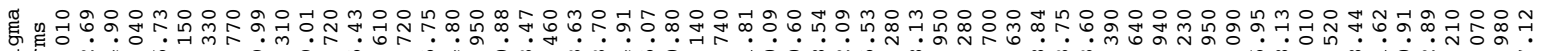

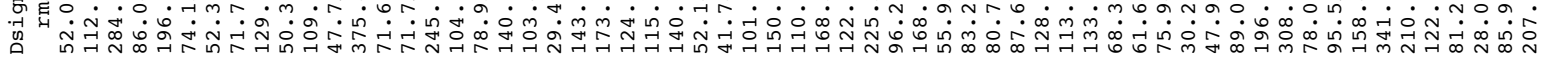

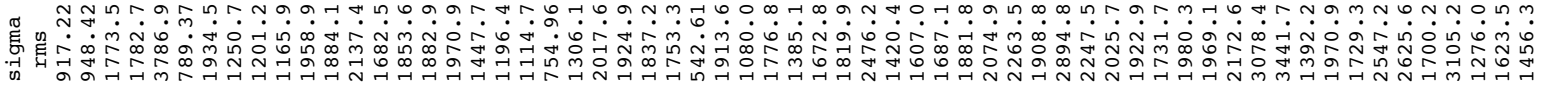

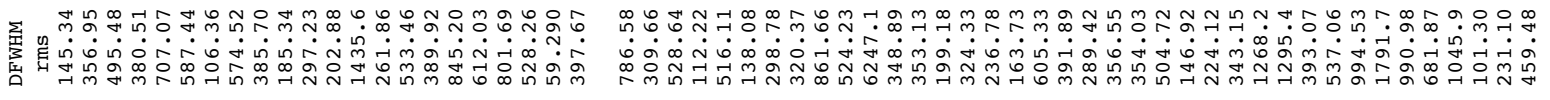

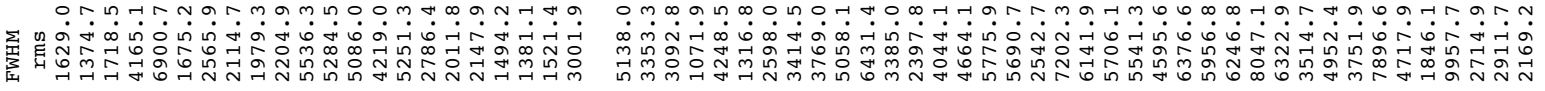

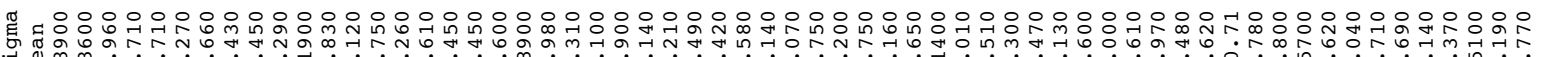

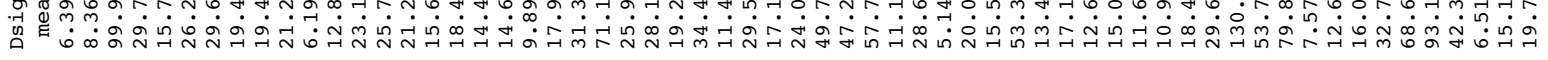

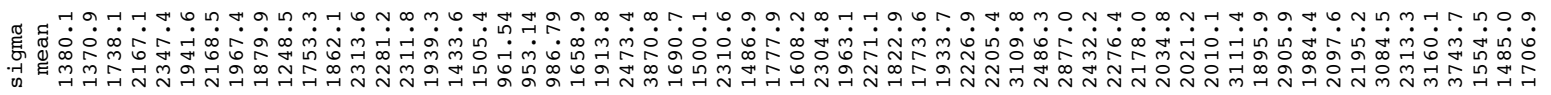

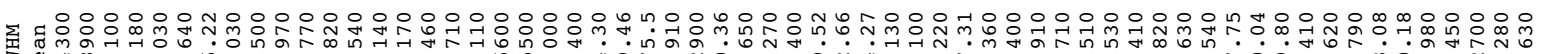

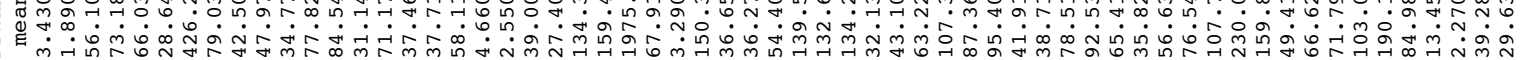

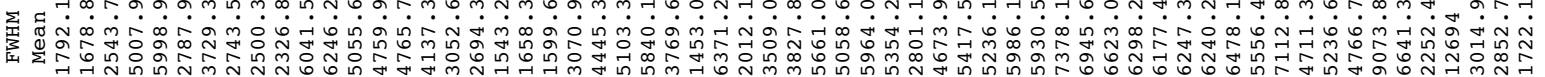

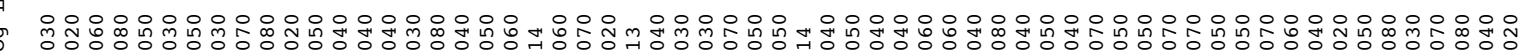
I

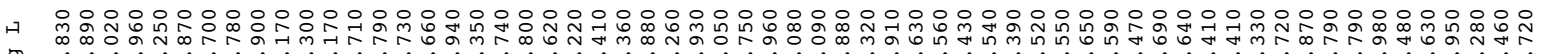

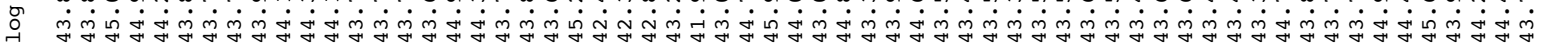

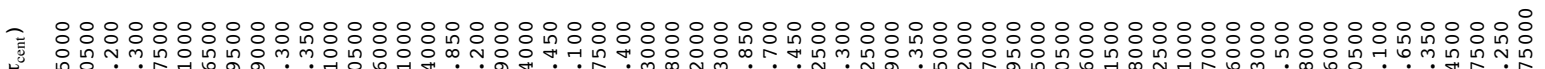

o

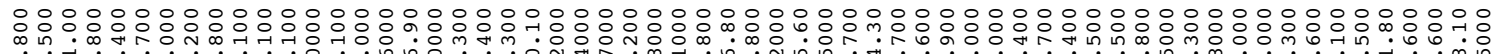
ف격

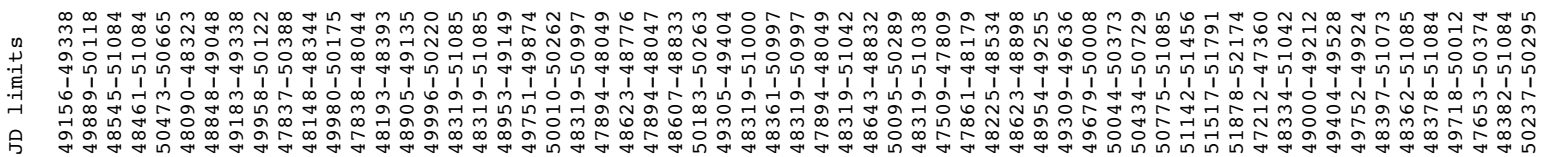

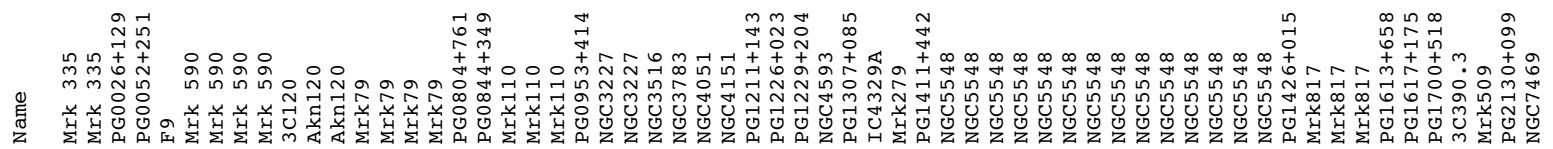

Fig. 3, is corresponds very roughly to a division around $\sigma_{\text {line }}=$ $2000 \mathrm{~km} \mathrm{~s}^{-1}$, which is approximately $F W H M=4000 \mathrm{~km} \mathrm{~s}^{-1}$; our demarcation is thus interestingly reminiscent of the division of AGNs by Sulentic et al. (2000) into a Population A, with $F W H M \leq 4000 \mathrm{~km} \mathrm{~s}^{-1}$, and a Population B, with $F W H M \geq$ $4000 \mathrm{~km} \mathrm{~s}^{-1}$. Sulentic et al. identify Population A, which includes NLS1s, with low BH mass and high accretion-rate sources, and Population B with low accretion-rate radio-loud (or pre-/post-cursors of radio-loud) sources. Apparently the large range of the line-width ratio $F W H M / \sigma_{\text {line }}$ is telling us something important about the BLR: the line-of-sight kinematics and/or the BLR structure show a great variety. The inclination can also play an important role, as will be discussed later.

\subsection{Effects other than inclination: the importance of NGC 5548}

The galaxy NGC 5548 is the best-studied of all reverberationmapped AGNs. In Figs. 1-3, NGC 5548 appears multiple times 

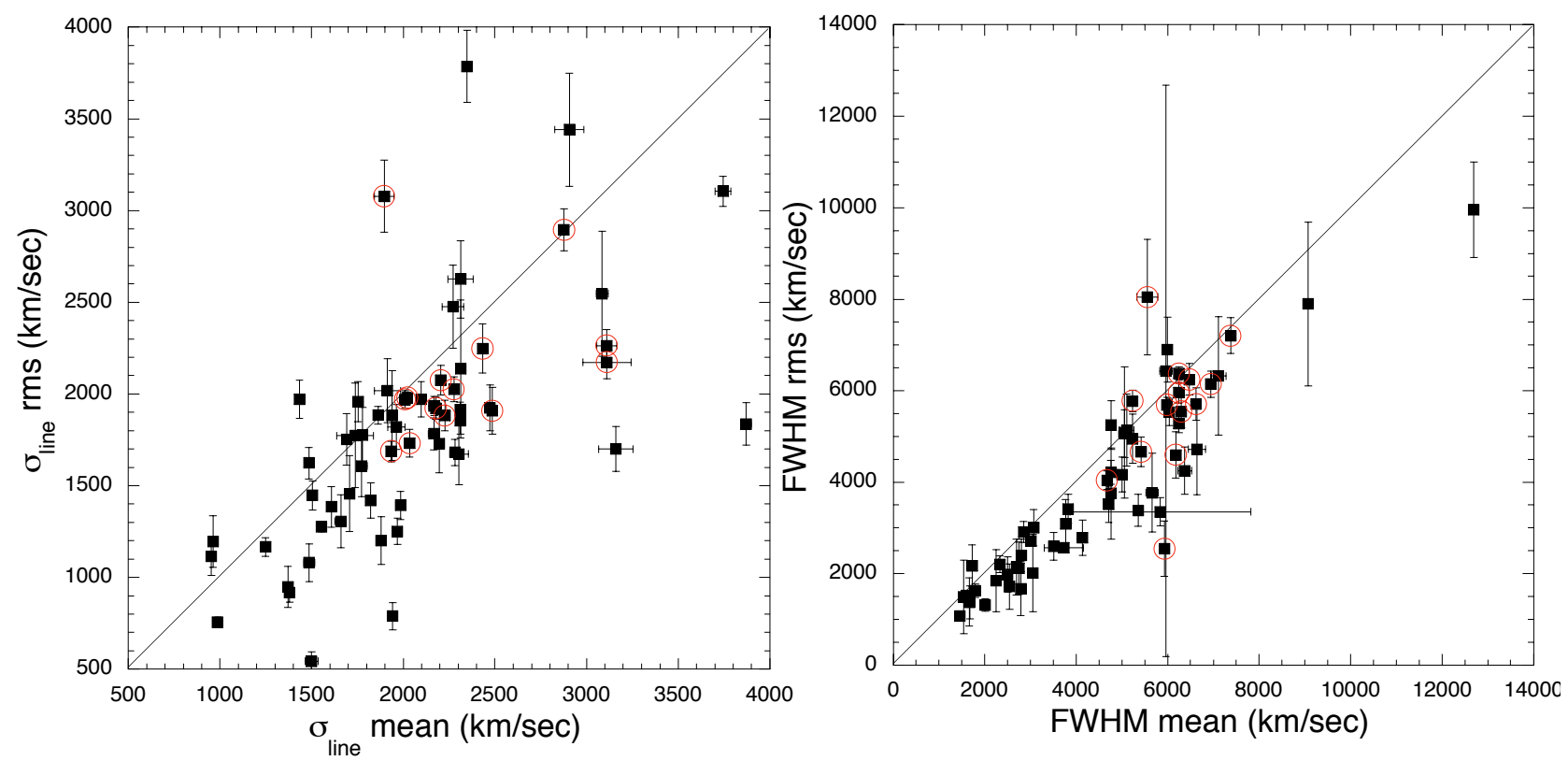

Fig. 1. The left panel shows $\sigma_{\text {line }}$ in the rms spectrum versus $\sigma_{\text {line }}$ in the mean spectrum for $\mathrm{H} \beta$ in all 35 datasets in Table 1 . The right panel shows the FWHM in the rms spectrum versus the FWHM in the mean spectrum. The open circles mark the different datasets of NGC 5548. The straight line traces the diagonal.
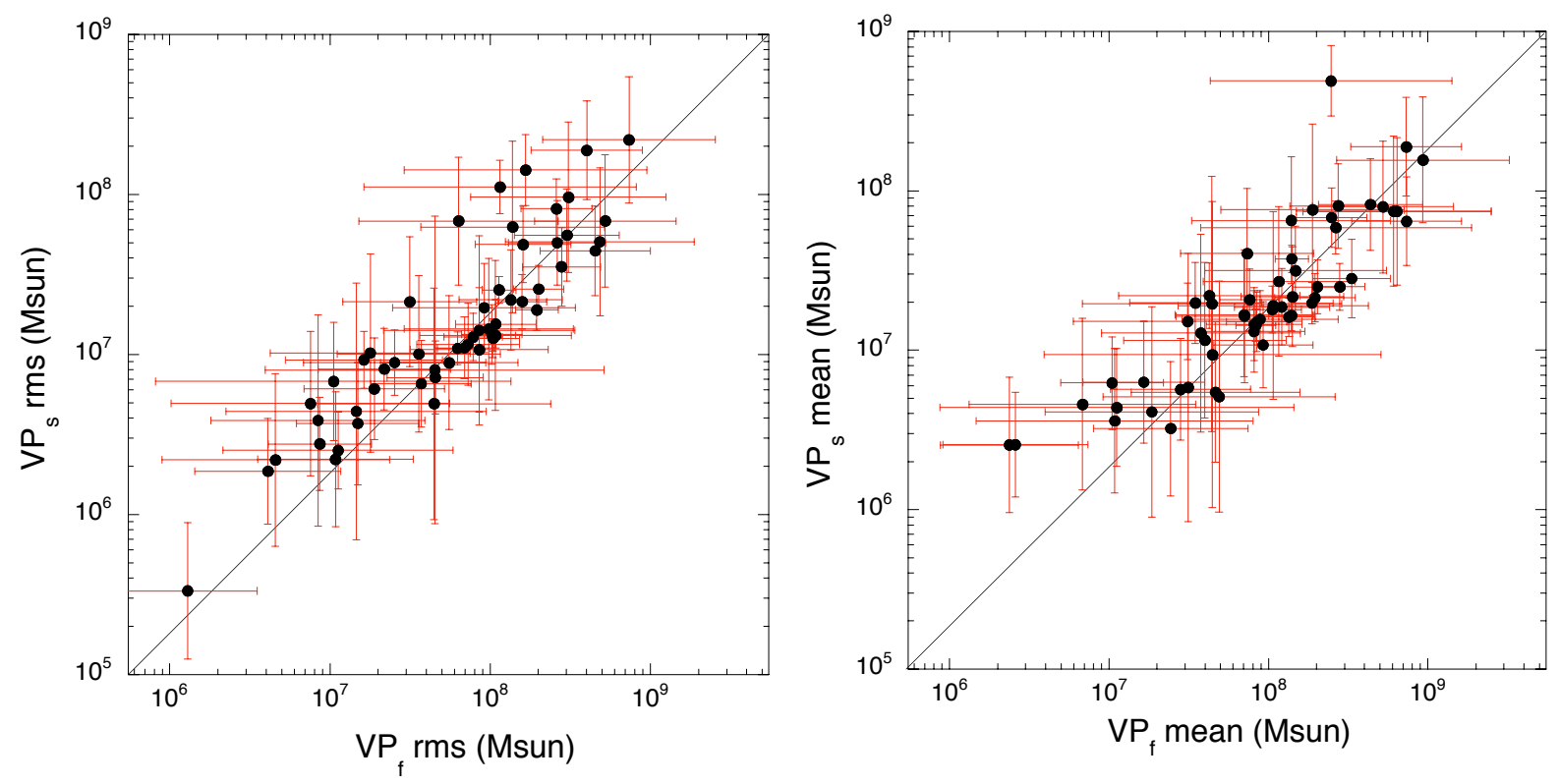

Fig. 2. Virial product based on $\sigma_{\text {line }}\left(V P_{\mathrm{s}}\right)$, versus the virial product based on the $F W H M\left(V P_{\mathrm{f}}\right)$, for all but four of the datasets (identified in the text) in Table 1, based on rms spectra (left panel) and mean spectra (right panel). The straight lines correspond to a Gaussian profile, for which $V P_{\mathrm{s}}=V P_{\mathrm{f}} / 5.52$.

as a result of 14 separate years of intensive optical spectroscopic monitoring first at the Wise Observatory in 1988 (Netzer et al. 1990) then for 13 consecutive years by the International AGN Watch beginning in late 1989 (Peterson et al. 2002 and references therein). We see that all the quantities in Table 1 vary with time: the FWHM in the mean spectrum and $\sigma_{\text {line }}$ in both the mean and the rms spectrum vary by less than a factor of two, but the FWHM in the rms spectrum varies by up to a factor of four. Figure 3 shows that $F W H M / \sigma_{\text {line }}$ can also vary by a factor larger than two, and moreover that it can migrate between our two arbitrarily defined populations, as well as those of Sulentic et al. (2000). This demonstrates clearly that $F W H M / \sigma_{\text {line }}$ does not depend solely on either mass or inclination, as these are constant over the timescales involved.

An obvious requirement is that the $\mathrm{BH}$ mass, or virial product, must be constant for all the individual datasets on NGC 5548. In Fig. 4, we show $V P_{\mathrm{s}}$ and $V P_{\mathrm{f}}$, as measured in the mean and rms spectra, as a function of the mean luminosity, for 13 NGC 5548 datasets $^{2}$. In this diagram, the optical continuum luminosity has been corrected for host-galaxy contamination by using the value of the starlight contribution given

${ }^{2}$ We have suppressed the point corresponding to the Wise Observatory data from 1988 which is not reliable on account of a variable line-spread function, as noted by Peterson et al. (2004). 


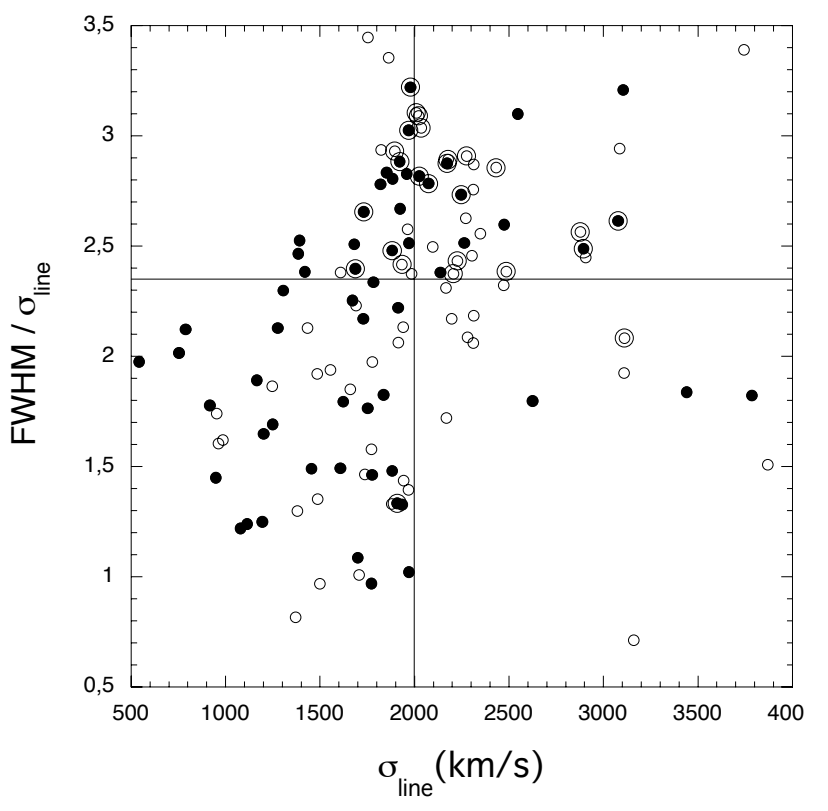

Fig. 3. The $\mathrm{H} \beta$ line-width ratio $F W H M / \sigma_{\text {line }}$ versus $\sigma_{\text {line }}$ in all the datasets in Table 1 . The open and filled circles correspond respectively to values based on mean and rms spectra. The large symbols mark the different datasets for NGC 5548. The horizontal line is the value of the ratio for a Gaussian profile, and the vertical line is an arbitrary division at $\sigma_{\text {line }}=2000 \mathrm{~km} \mathrm{~s}^{-1}$. The horizontal line divides the samples into our Populations 1 (lower) and 2 (upper), and the vertical line approximates the division of Sulentic et al. (2000) into Populations A (left) and B (right).

by Bentz et al. (2006). Despite the large variations in the value of $\sigma_{\text {line }}$ as seen in Fig. 1, we see that all the measures of $V P_{\mathrm{s}}$ in both the mean and rms spectra are consistent with a constant value, for masses based on either the mean or rms spectra. The discrepant point is from Year 12 (2000) of the AGN Watch program (Peterson et al. 2002), which was the most poorly sampled $\mathrm{H} \beta$ light curve in the whole series and yielded somewhat ambiguous cross-correlation results (see Fig. 2 of Peterson et al. 2002).

\subsection{Comparison with masses based on $\sigma_{*}$}

Ferrarese \& Merritt (2000) and Gebhardt et al. (2000) showed that a tight relationship exists between the $\mathrm{BH}$ mass $M_{\mathrm{BH}}$ and the velocity dispersion $\sigma_{*}$ of the stars in the bulge of the host galaxy. The galaxies that define the $M_{\mathrm{BH}}-\sigma_{*}$ relationship are nearly all ${ }^{3}$ quiescent galaxies whose $\mathrm{BH}$ masses were measured by stellar or gas kinematics. It is difficult, but possible, to measure bulge velocity dispersions in at least the lowest-luminosity AGNs. At the present time, velocity dispersion measurements have been published for about 16 AGNs, i.e., almost half of the reverberation-mapped sample. Onken et al. (2004) plotted the values of $V P_{\mathrm{s}}$ versus $\sigma_{*}$ for these objects and obtained a relationship consisent with the quiescent-galaxy $M_{\mathrm{BH}}-\sigma_{*}$ relationship. By making the assumption that the AGN $M_{\mathrm{BH}}-\sigma_{*}$ relationship is identical to that for quiescent galaxies, they were able to convert the $V P_{\mathrm{s}}-\sigma_{*}$ relationship to a $M_{\mathrm{BH}}-\sigma_{*}$ relationship by determining a statistical value for the scale factor of Eq. (1). Onken et al. found $\langle f\rangle=5.5 \pm 1.8$.

\footnotetext{
3 One Seyfert 2 galaxy, NGC 4258, whose BH mass was measured by megamaser motions (Miyoshi et al. 1995), was included in the sample of galaxies that defined the $M_{\mathrm{BH}}-\sigma_{*}$ relationship.
}

Here we will carry out a similar exercise, but for all four ways of computing the virial product, i.e. $V P_{\mathrm{s}}$ and $V P_{\mathrm{f}}$ for both the mean and rms spectra. We compute for each AGN in the Onken sample an estimate of the $\mathrm{BH}$ mass $M_{\sigma *}$ in solar masses from the formula of Tremaine et al. (2002),

$\log M_{\sigma *}=8.13+4.02 \log \left(\sigma_{*} / 200 \mathrm{~km} \mathrm{~s}^{-1}\right)$.

We have excluded two objects with highly uncertain reverberation-based masses, IC 4329A and NGC 4593, leaving a sample of 14 objects and 39 datasets (herafter the "Onken sample").

In Table 2, we give the average scale factors, computed as by Onken et al. (2004). We first note that the scale factor for the entire sample using $\sigma_{\text {line }}$ measured the rms spectra yields a value identical to that obtained by Onken et al., $f=5.5$. We also note that using $\sigma_{\text {line }}$ measured the mean spectra yields $f=$ 3.85 , reflecting our earlier observation that on average $\sigma_{\text {line }}$ is $\sim 20 \%$ broader in the mean spectra than in the rms spectra; the scale factor based on $F W H M$ is only $20 \%$ larger for the mean spectra compared to the rms spectra, however, because FWHM is typically only about $10 \%$ broader in the mean spectra than in the rms spectra. We see also that $V P_{\mathrm{f}} / V P_{\mathrm{s}} \approx 4$, as expected since $\left\langle F W H M / \sigma_{\text {line }}\right\rangle \approx 2$.

As noted earlier, there is great range in the values of $F W H M / \sigma_{\text {line }}$ and thus the scale factors that we would compute will be strongly dependent on the value of this ratio for the objects in the sample. This leads us to compute the scale factors separately, again for all four ways of computing the virial product, for our two populations, Population 1 with $F W H M / \sigma_{\text {line }}<$ 2.35 and Population 2 with $F W H M / \sigma_{\text {line }}>2.35$. These values are also given in Table 2. We have also computed separate scale factors for Population A $\left(F W H M<4000 \mathrm{~km} \mathrm{~s}^{-1}\right)$ and Population B $\left(F W H M>4000 \mathrm{~km} \mathrm{~s}^{-1}\right)$ of Sulentic et al. (2000). There are 9 objects in the Onken sample in Population 1, among which the 6 objects of Population A are all included. The 6 common objects are NGC 4051, NGC 3783, NGC 7469, Mrk 110, Mrk 590, and 3C 120. Population B consists of 8 objects, among which the 5 objects of Population 2 are all included, namely, NGC 4151, NGC 5548, Mrk 817, Akn 120, and 3C 390.3.

The results of this exercise are very revealing: there is a clear difference between the scale factors of the different subsets, especially if we compare the objects common to Populations 1 and $\mathrm{A}$ with those common to Populations 2 and B. For $V P_{\mathrm{f}}$, Populations 1 and $\mathrm{A}$ have scale factors larger by a factor of $\sim 3$ than those for Populations 2 and B, both in the mean and rms spectra. This trend does not exist for $V P_{\mathrm{s}}$; in both the mean and rms spectra, the scaling factors computed from $V P_{\mathrm{s}}$ are consistent with a constant value. It is clear that the statistics are poor, and that more objects in the sample are urgently needed. Nevertheless some conclusions can already be drawn from these results:

1. Since scale factors based on $\sigma_{\text {line }}$ for different populations are consistent with a single value, contrary to the case for scale factors based on $F W H M, V P_{\mathrm{s}}$ is not sensitive to the line profile, the Eddington ratios, inclination, or whatever factors distinguish between Populations 1 and 2 or A and B, whereas $V P_{\mathrm{f}}$ clearly is affected by these factors. In other words, $\sigma_{\text {line }}$ is a less biased measure of the velocity dispersion than is FWHM.

2. Use of $F W H M$ as line-width measure and applying a constant scale factor corresponding to the average for the whole sample will underestimate the masses of Populations 1 and $A$ (including NLS1s) and overestimate the masses of 

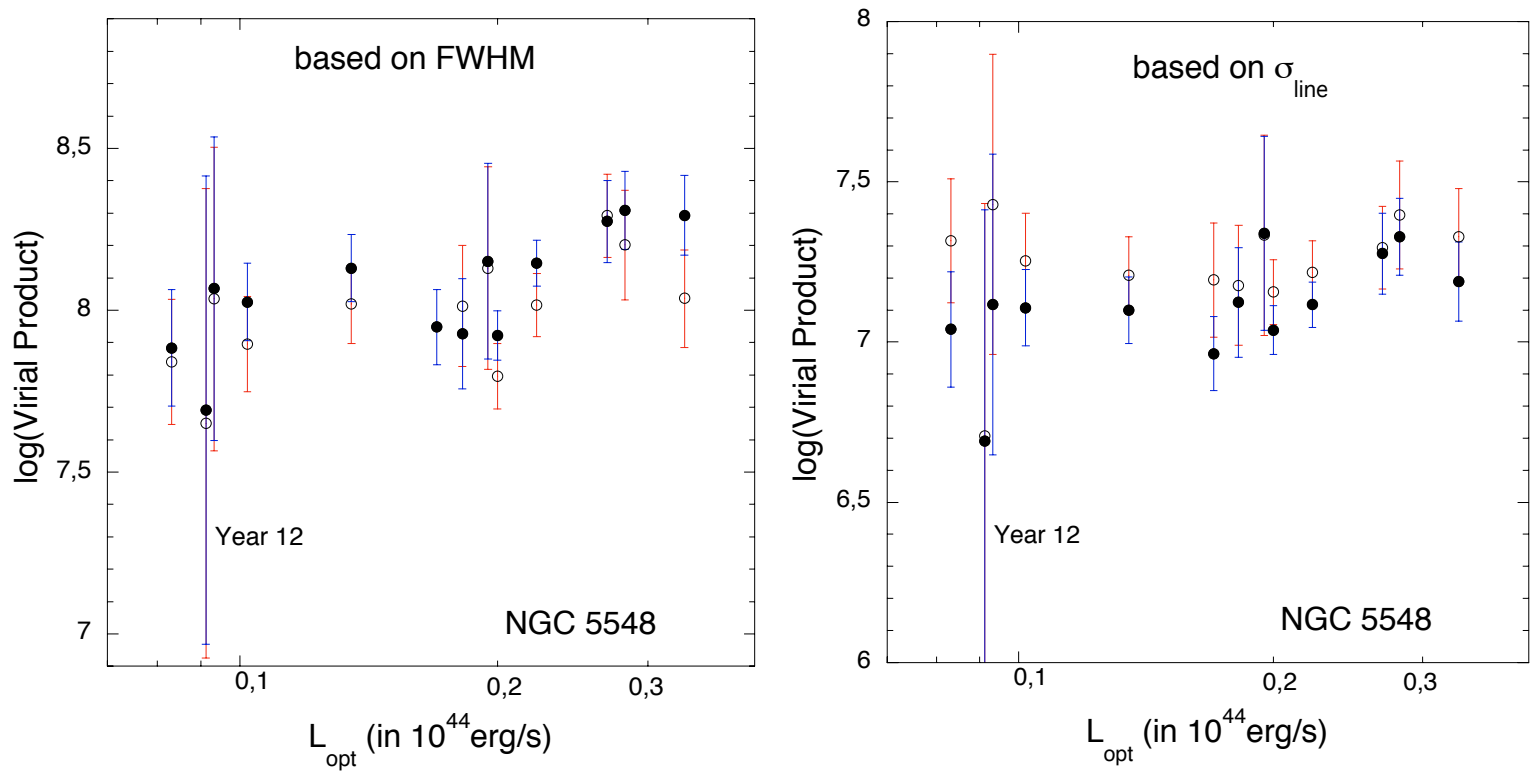

Fig. 4. The virial product based on the FWHM (left panel) and on $\sigma_{\text {line }}$ (right panel), versus the optical luminosity of NGC 5548, corrected for the host-galaxy starlight contribution. The open and filled circles correspond respectively to values based on the mean and rms spectra. In the left panel, the point corresponding to the rms spectrum of Year 5 has been suppressed as determination of the $F W H M$ of $\mathrm{H} \beta$ is problematic in this case (see Fig. 14 of Peterson et al. 2004).

Table 2. The scale factors with their uncertainties for the Onken sample and for two populations (1) separated at $F W H M / \sigma_{\text {line }}=2.35$ (Pop1 and Pop2) as explained in the text and (2) separated at $F W H M=$ $4000 \mathrm{~km} \mathrm{~s}^{-1}$ (PopA and PopB) according to Sulentic et al. (2000).

$$
f\left(\sigma_{1 \text { ine }}\right) \quad d f\left(\sigma_{1 \text { ine }}\right) \quad f(\text { FWHM }) \quad d f(\text { FWHM })
$$

MEAN SPECTRUM

$\begin{array}{lllll}\text { total } & 3.85 & 1.15 & 1.17 & 0.50 \\ \text { Pop1 } & 4.20 & 2.09 & 1.81 & 1.38 \\ \text { Pop2 } & 3.48 & 1.09 & 0.69 & 0.19 \\ \text { PopA } & 3.93 & 1.97 & 2.12 & 1.47 \\ \text { PopB } & 3.75 & 1.13 & 0.52 & 0.13\end{array}$

RMS SPECTRUM

$\begin{array}{lllll}\text { total } & 5.49 & 1.65 & 1.44 & 0.49 \\ \text { Pop1 } & 5.36 & 2.71 & 2.21 & 1.22 \\ \text { Pop2 } & 5.66 & 1.49 & 0.92 & 0.27 \\ \text { PopA } & 6.23 & 3.47 & 2.53 & 1.49 \\ \text { PopB } & 4.73 & 1.11 & 0.81 & 0.19\end{array}$

Populations 2 and $B$. This could be a contributing factor to why high ratios of bulge-to-BH masses have been found in NLS1s (Wandel 2001; Mathur et al. 2001), since the BH masses are based on FWHM in these works. On the other hand, since it appears that the $F W H M$ is influenced by some still undefined physical parameters to which $\sigma_{\text {line }}$ is not sensitive, it could be used as an indicator of whatever these currently unidentified parameters turn out to be. We argue below that one unidentified parameter is BLR inclination, and another is the Eddington ratio.

3. It is possible to correct $V P_{\mathrm{f}}$ to obtain an unbiased $\mathrm{BH}$ mass. At the present time, the correction is only approximate because the uncertainties on the scale factors are large. According to Table 2, a simple prescription is to use $f=2.35$ for Population 1 and $f=0.85$ for Population 2 for the rms spectrum, and $f=1.5$ for Population 1 and $f=0.50$ for Population 2 for the mean spectrum, so the mass ${ }^{4}$ is given by $M_{\mathrm{BH}}=f V P_{\mathrm{f}}$. But to avoid a discontinuity between these two rather arbitrarily defined populations, we tentatively suggest using for the rms spectrum,

$$
\begin{aligned}
f= & 2.35 \quad\left(\text { for }\left(F W H M / \sigma_{\text {line }}\right) \leq 1.4\right) \\
= & 3.85-1.07\left(F W H M / \sigma_{\text {line }}\right) \\
& \quad\left(\text { for } 1.4<\left(F W H M / \sigma_{\text {line }}\right)<2.8\right) \\
= & 0.85 \quad\left(\text { for }\left(F W H M / \sigma_{\text {line }}\right) \geq 2.8\right),
\end{aligned}
$$

and for the mean spectrum,

$$
\begin{aligned}
f= & 1.5 \quad\left(\text { for }\left(F W H M / \sigma_{\text {line }}\right) \leq 1.4\right) \\
= & 2.5-0.71\left(F W H M / \sigma_{\text {line }}\right) \\
& \quad\left(\text { for } 1.4<\left(F W H M / \sigma_{\text {line }}\right)<2.8\right) \\
= & 0.5 \quad\left(\text { for }\left(F W H M / \sigma_{\text {line }}\right) \geq 2.8\right) .
\end{aligned}
$$

It is rarely the case, however, that $\sigma_{\text {line }}$ measurements are available, especially in published compilations of line widths. in such cases, we suggest using for the rms spectrum,

$$
\begin{aligned}
f= & 2.35 \quad\left(\text { for }\left(F W H M \leq 2000 \mathrm{~km} \mathrm{~s}^{-1}\right)\right. \\
= & 3.1-1.5\left(F W H M / 4000 \mathrm{~km} \mathrm{~s}^{-1}\right) \\
& \quad\left(\text { for } 2000<F W H M<6000 \mathrm{~km} \mathrm{~s}^{-1}\right) \\
= & 0.85 \quad\left(\text { for } F W H M \geq 6000 \mathrm{~km} \mathrm{~s}^{-1}\right),
\end{aligned}
$$

and for the mean spectrum,

$$
\begin{aligned}
f= & 1.5 \quad\left(\text { for }\left(F W H M \leq 2000 \mathrm{~km} \mathrm{~s}^{-1}\right)\right. \\
= & 2-\left(F W H M / 4000 \mathrm{~km} \mathrm{~s}^{-1}\right) \\
& \quad\left(\text { for } 2000<F W H M<6000 \mathrm{~km} \mathrm{~s}^{-1}\right) \\
= & 0.5 \quad\left(\text { for } F W H M \geq 6000 \mathrm{~km} \mathrm{~s}^{-1}\right) .
\end{aligned}
$$

${ }^{4}$ It is perhaps interesting that prior to the actual calibration of the reverberation-based mass scale by Onken et al. (2004), it was common to assume $f=0.75$ (Netzer 1990; Wandel et al. 1999; Kaspi et al. 2000) for $F W H M$-based mass estimates. 

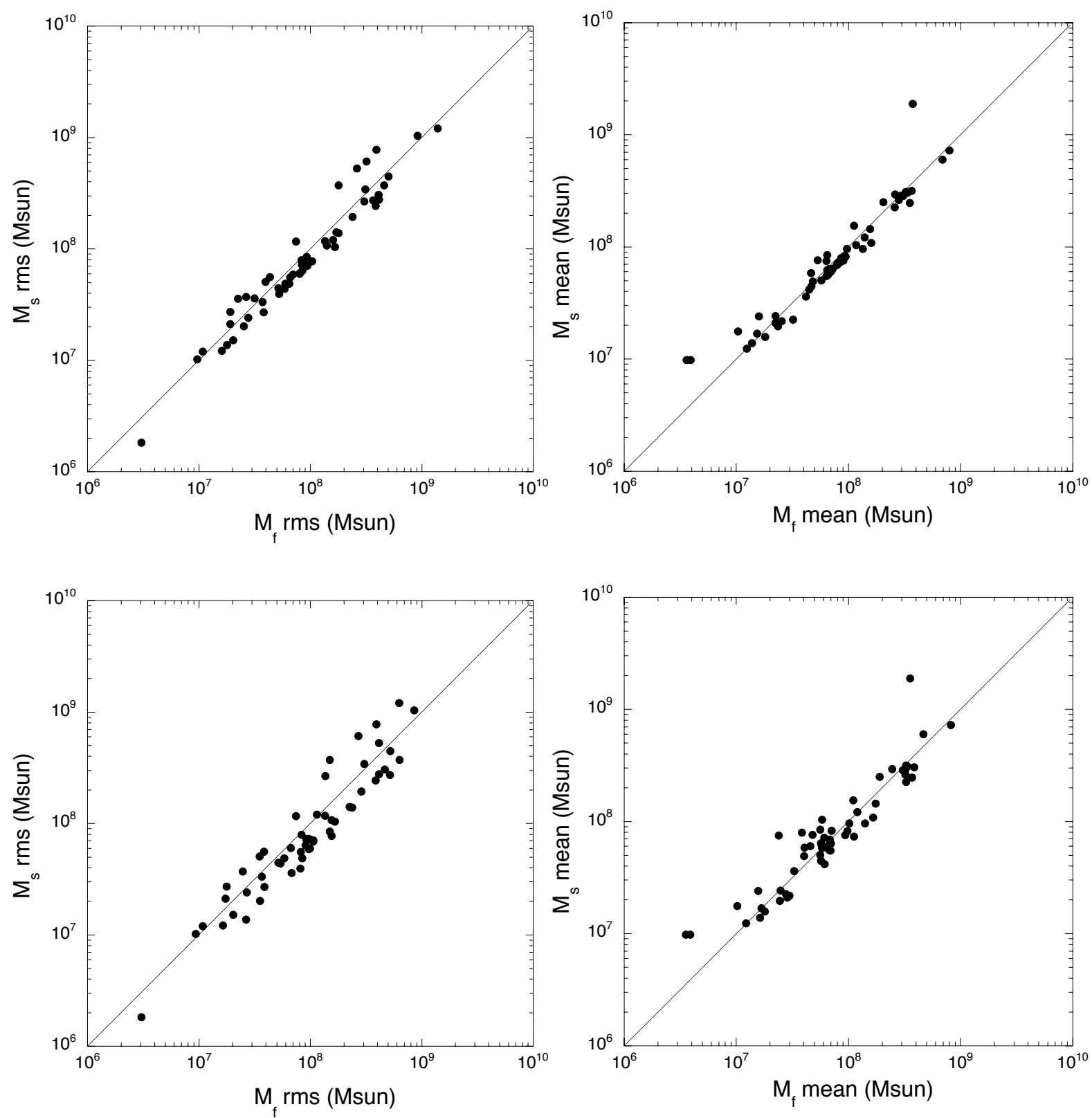

Fig. 5. Mass estimates based on $\sigma_{\text {line }}$ as a line-width measure are plotted versus masses based on $F W H M$ measurements and adjusted according to Eqs. (4) and (5) (upper panels) and to Eqs. (6) and (7) (lower panels). The data in the left panels are based on line widths in the rms spectrum and those based on the mean spectrum are shown in the right panels. The same four datasets as in Fig. 2 have been suppressed. The straight lines trace the diagonals.

For $\sigma_{\text {line-based data, for all AGNs we suggest using the value }}$ $f=5.5$, (i.e., $M_{\mathrm{BH}}=5.5 V P_{\mathrm{s}}$ ) for the rms spectrum, as proposed by Onken et al. (2004), and $f=3.85$ (i.e. $M_{\mathrm{BH}}=3.85 V P_{\mathrm{s}}$ ) for the mean spectrum.

\subsection{Application of the scale factors: a consistency test}

In the last section, we provided scaling factors to convert both $V P_{\mathrm{s}}$ and $V P_{\mathrm{f}}$ into $\mathrm{BH}$ masses. We now apply these to the entire sample of 35 objects from Table 1 .

Figure 5 compares the $\sigma_{\text {line }}$-based masses, computed using $f=5.5$ and $f=3.85$ for the rms and mean spectra, respectively, with the corrected FWHM-based masses in both the mean and rms spectrum for all the data sets in the sample. In the upper panels, the $F W H M$-based masses are corrected by using Eqs. (4) and (5), while in the lower panels, the corrections are given by Eqs. (6) and (7). The two formulations give very similar results, and the scatter is reduced compared to Fig. 2, even when only the FWHM is used for the correction. This demonstrates the utility of using Eqs. (6) and (7) to adjust $F W H M$-based mass estimates when $\sigma_{\text {line }}$ is not known.

\subsection{Line width measures and the Eddington ratio}

The line-width ratio $F W H M / \sigma_{\text {line }}$ is of potential importance as it may trace physical parameters in the inner regions of AGNs. Since we have already pointed out a qualitative correlation with Eigenvector 1 properties, which has been argued to measure the Eddington ratio (e.g., Boroson 2002), it is now of interest to look at this more quantitatively. In Fig. 6, we show the line-width 


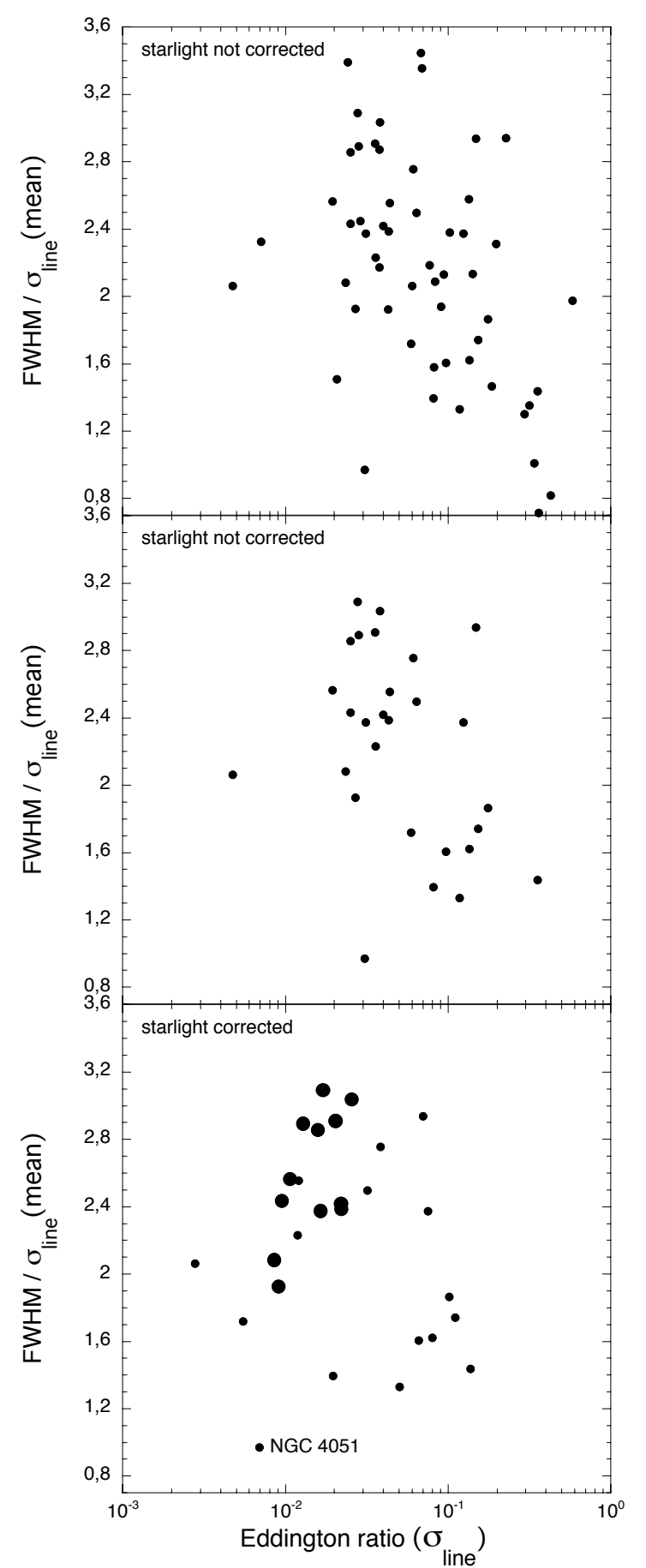

Fig. 6. The line-width ratio $F W H M / \sigma_{\text {line }}$ for $\mathrm{H} \beta$ in the mean spectra, versus the Eddington ratio (based on masses from Peterson et al. 2004) for all the datasets for the 35 reverberation-mapped objects in Table 1 (top panel). The middle panel shows the subset of points from the top panel which can be corrected for the host-galaxy starlight contribution to the luminosity using values from Bentz et al. (2006). The bottom panel shows the points from the middle panel after correction for starlight. The larger points in the lower panel represent independent data sets on NGC 5548. Assumptions used in computing the Eddington ratio are described in the text.

ratio $F W H M / \sigma_{\text {line }}$ as measured in the mean spectrum (since it is less noisy than the rms spectrum) as a function of Eddington ratio, which has been computed assuming that the bolometric luminosity is $L_{\mathrm{bol}} \approx 10 L_{\mathrm{opt}}$ (based loosely on Kaspi et al. 2000 and Elvis et al. 1994), and $M_{\mathrm{BH}}$ is based on measurements of $\sigma_{\text {line }}$ in the rms spectrum, which appears to yield the most accurate mass estimate (Peterson et al. 2004). The top panel of Fig. 6 shows a clear anticorrelation between the line-width ratio $F W H M / \sigma_{\text {line }}$ and the Eddington rate, though with considerable scatter. The dependency of $F W H M / \sigma_{\text {line }}$ on Eddington rate is physically quite plausible as we expect that the structure and the dynamics of the BLR, which determine the line profile, depend on the accretion rate. We note, however, that the Eddington rates in this figure are somewhat overestimated because the optical luminosities have not been corrected for contamination by host-galaxy starlight. At the present time, it is possible to accurately correct for the host-galaxy contribution to the luminosity for only a subset of these AGNs, those observed by Bentz et al. (2006). In the middle panel of Fig. 6, we show the subset of points from the top panel for which Bentz et al. provide measurements of the starlight contribution. In the bottom panel, we show the points from the middle panel after correction for starlight. The anticorrelation that is clearly seen in the top panel appears to have vanished in the middle and bottom panels. This is simply because most the highEddington rate objects are PG quasars which were not observed by Bentz et al. Given the higher luminosities of these sources, the starlight corrections are likely to be small so the points in the lower right of the top panel will have very nearly the same positions in the bottom panel, thus preserving the anticorrelation. In the lower panel, we highlight by use of larger symbols the multiple independent measurements of NGC 5548. These are dispersed in a direction almost normal to the anticorrelation seen in the top panel, which suggests that much of the scatter in this relationship can be attributed to intrinsic variability. We will discuss this further in a future paper.

To summarize this section, we have shown that we can crudely separate AGNs into high Eddington ratio objects whose spectra are characterized by small values of $F W H M / \sigma_{\text {line }}$ (Population 1) and low Eddington ratio objects, which have large values of $F W H M / \sigma_{\text {line }}$ (Population 2). We find that $\sigma_{\text {line }}$ is an unbiased estimator of the $\mathrm{BH}$ mass, whereas $F W H M$ requires an adjustment for its sensitivity to still undefined physical parameters, likely to be the Eddington ratio and/or the source inclination. Finally, we note that $F W H M / \sigma_{\text {line }}$ is not correlated with $\mathrm{BH}$ mass, or with luminosity.

\section{Influence of the BLR inclination}

Since we observe only the line-of-sight velocity distribution of the BLR, it is obvious that inclination of the BLR will play a significant role in the value of the scale factor except in the unlikely case of an isotropic velocity field. For instance, Krolik (2001) pointed out that if the BLR is a thin disk, it would lead to an underestimation of the mass by one or two orders of magnitude for objects seen at low inclination. To study the influence of the inclination, it is necessary to have an idea of the structure and the dynamics of the BLR. In this section, we show that a few simple but plausible structures can be parameterized in a common fashion and we explore the effects of inclination in this context as an exercise.

\subsection{Structure and dynamics of a rotationally dominated BLR}

It is often noted that the structure and the dynamics of the BLR are not known, despite more than thirty years of intense studies. Indeed we only know with certainty, thanks to reverberation studies, that the region emitting the "low-ionization lines" like $\mathrm{H} \beta$ is gravitationally bound to the $\mathrm{BH}$ (Peterson \& Wandel 1999, 2000; Onken \& Peterson 2002; Kollatschny 2003a) and more 
precisely that the bulk radial velocity is small (Clavel 1991). But it remains true that we do not know if the BLR is a spherical structure dominated by turbulent motions, if it includes a wind component, or if it is a disk dominated by rotational motions,

There is actually fairly strong evidence for the latter case in radio-loud AGNs. Based on the ratio of the radio core flux to the extended radio lobe flux, $R$, which is related to the relativistic amplification of the core source and is large when the object is seen face-on, Wills \& Browne (1986) and Jackson \& Browne (1991) found a lack of broad lines for face-on objects. Also, Vestergaard et al. (2000) find that the width of the base of the C IV $\lambda 1549$ emission line is inversely correlated with $R$, suggesting the existence of a largely radial disk wind. In superluminal objects where the inclination can be derived quite precisely, Rokaki et al. (2003) have shown that the line width is anti-correlated with several beaming indicators, and is consistent with a disk structure of the BLR. Finally, double-peaked profiles of the Balmer lines, though observed only in a small fraction of radio AGNs, are another suggestion of disk structure (Eracleous \& Halpern 1994 and subsequent papers). Such double peaks, more or less pronounced, are characteristics of our Population 2 AGNs.

There is no direct evidence that this picture also applies to radio-quiet objects, but several observations fit this model quite well (e.g., see Smith et al. 2005). There is also indirect evidence which cannot be ignored. A common view of the BLR is that it consists of a large number of clouds with high velocities surrounding a central source of UV-X-ray radiation that photoionizes the clouds and produces line emission by reprocessing. These clouds should have a large covering factor, since at least $10 \%$ of the central X-UV source, and in some cases a larger fraction, has to be absorbed by the BLR in order to account for the large measured equivalent widths of the broad lines. On the other hand, the column density of the clouds is inferred to be high, $10^{23-24} \mathrm{~cm}^{-2}$. There is actually no observational limit to the real column density of the clouds, as a large fraction can stay neutral and unobservable (for a review, see Collin-Souffrin \& Lasota 1988, for example). The lack of Lyman edges in absorption and of damped Ly $\alpha$ lines in AGN spectra is difficult to explain in this context, unless the BLR clouds are not located on the line of sight to the central source. Such a configuration is natural in the framework of unified schemes (Antonnucci \& Miller 1985), if the BLR has a disk structure in the same plane as that of the obscuring "torus" and of the inner disk. Since for Seyfert 1 galaxies the central continuum is seen from above at relatively small inclinations to the disk normal, it would not be absorbed by this BLR disk.

As explained above, the BLR disk must "see" the central source, as it must be able to capture a large fraction of its ionizing photons. We can thus immediately eliminate the possibility that the BLR disk is a thin, flat structure that is directly illuminated by the central UV-X-ray source. In this scenario, the central source should have a large scale height above the disk, of the order the radius of the BLR disk itself. But we know from the study of the continuum emission, in particular from the correlations between the UV and X-ray flux variability, that the UV-X-ray source is located much closer to the $\mathrm{BH}$ than the BLR, typically at distances of 10 to $10^{2} R_{\mathrm{G}}$, where $R_{\mathrm{G}}$ is the gravitational radius, while the distance of the BLR is of the order of $10^{3}$ to $10^{4} R_{\mathrm{G}}$. A possible exception could be the case of low-luminosity AGNs with very broad double-peaked profiles, where it has been suggested that the inner part of the disk might be an inflated ion-supported torus (cf. Sect. 4).
Another possibility is that the surface of the BLR disk is illuminated indirectly by the central source, which could occur if its radiation is backscattered by a hot medium (or corona) located above the BLR disk, as proposed by Dumont \& Collin-Souffrin (1990). This model faces some difficulties, however, as the hot medium should have a Thomson thickness at least of the order of unity. In this case, the variations of the central source would be smoothed by multiple electron scattering, and the shape of the observed X-ray continuum would be modified by absorption and Compton diffusion (Reynolds \& Wilms 2000), two predictions that are contradicted by the observations. However the recent observations of very thick X-ray winds in some NLS1s (Pounds et al. 2003) and the suggestion by Gierlinski \& Done (2004) and Chevallier et al. (2005) that the soft X-ray excess is due to the absorption by a very thick warm absorber, could perhaps rehabilitate this idea for high accretion-rate objects. But it cannot be considered as a general solution for the BLR of all AGNs.

Thus it is unlikely that the BLR disk is a very thin flat structure entirely dominated by rotation, and other possibilities must be considered ${ }^{5}$.

\subsubsection{The BLR as a flared disk}

The required illumination of the BLR implies that the opening angle of the BLR disk should be large, i.e., $\Omega / 4 \pi \geq 0.1$. In other words, the BLR disk should have an aspect ratio larger than $H / R=0.1$ at the location of the line-emitting region, where $H$ is the disk thickness at the radius $R$. Moreover it should be "flared," i.e., that $H$ increases more rapidly than linearly with increasing $R$.

Such flared disks are predicted in the context of the "standard model" (Shakura \& Sunyaev 1973), and were invoked by Dumont \& Collin-Souffrin (1990) as being the origin of the lowionization lines in AGNs. In this case, the BLR disk should be sustained vertically by a pressure which is most probably provided by a turbulent velocity of the order $V_{\mathrm{Kep}} H / R$, where $V_{\mathrm{Kep}}$ is the local Keplerian velocity at the distance $R$. In this model, the observed value of $\Delta V_{\mathrm{obs}}$ is given by

$\Delta V_{\mathrm{obs}} \approx V_{\mathrm{Kep}}\left[(H / R)^{2}+\sin ^{2} i\right]^{1 / 2}$

where $i$ is the inclination of the system to our line of sight.

\subsubsection{The BLR as a warped disk}

A configuration which could be invoked for effective illumination of the disk by the central source is a warped disk structure. Such a structure is observed in the case of NGC 4258 through the maser spots (Greenhill et al. 1995; Miyoshi et al. 1995). In this object, the rotation law at the distance of the megamaser sources is Keplerian, but the disk is tipped downward by about $10^{\circ}$, which allows more of the disk surface to be directly exposed to the central source. Wijers \& Pringle (1999) have suggested that similar warping should arise in AGNs as a response of the disk to the radiation force from the central source, which can cause the disk to tilt out of the orbital plane and to precess. However, NGC 4258 is a low-luminosity AGN, and it is not clear that this would be the case in more luminous objects, which in particular would be more sensitive to the gravitational instability.

\footnotetext{
5 It is worthwhile recalling that when we speak of a "disk," it does not necessarily mean a continuous medium, but could refer to a clumpy medium made of an ensemble of clouds.
} 
In this model, the velocity is quasi-Keplerian at any radius, so $\Delta V_{\text {obs }}$ is expressed as

$\Delta V_{\mathrm{obs}} \approx V_{\mathrm{Kep}} \sin i$,

but the inclination $i$ varies with the radius, i.e., with the particular line under consideration. Another difference from a flat thin disk is that the line-emitting region extends over a range of radii, therefore over a range of inclinations. There is thus a lower limit of the inclination, $\Delta i$, while it can be arbitrarily small for a flat disk.

\subsubsection{A two-component BLR: a disk and a wind}

In recent years, arguments supporting the presence of disk winds have won some popularity on account of the ability of such structures to explain a number of observed phenomena such as X-ray and UV absorption, line emission, reverberation results, and some differences among Seyfert galaxies, quasars, broad-line radio galaxies, and the presence or absence of double-peaked emission-line profiles. The importance of outflows that are commonly seen in AGNs as "warm absorbers" in X-rays have been recognized through observations made with XMM-Newton and Chandra X-Ray Observatory. It has been suspected for a long time that the BLR has two components, one that is disk-like and other that is some kind of outflow, probably a disk wind, either magnetohydrodynamically or radiatively driven. The wind scenario also obviates the problem of having to confine the lineemitting clouds. Key papers on the topic of disk winds are by Murray \& Chiang $(1995,1998)$ and by Proga \& Kallman (2004). In this model, the broad emission lines are emitted from the base of the disk winds (see also Elvis 2000).

A plausible configuration is thus a BLR made of two dynamically distinct components, a disk and a wind. The velocity dispersion could be written as

$\Delta V_{\mathrm{obs}} \approx\left[\alpha^{2}\left(a^{2}+\sin ^{2} i\right) V_{\mathrm{Kep}}^{2}+\beta^{2} V_{\mathrm{out}}^{2} \cos ^{2} i\right]^{1 / 2}$,

where $V_{\text {out }}$ is the outflow velocity, assumed to be normal to the disk, and $\alpha$ and $\beta$ are the contributions of the thick disk and of the wind, respectively.

However, the $V_{\text {out }} \cos i$ term in Eq. (10) cannot typically dominate the $\mathrm{H} \beta$ line, or the $\mathrm{H} \beta$ wing would display a strong asymmetry due to absorption on the receding part of the wind located on the far side of the accretion disk relative to the central source as seen by the observer. This is however observed in the most extreme objects of Population A which also show an extended blue wing in the high-ionization line C IV $\lambda 1549$ (cf. Sulentic et al. 2000), or in NLS1s which also show a blue wing in the [O III] $\lambda \lambda 4959,5007$ lines (cf. Zamanov et al. 2002; Aoki et al. 2005; Boroson 2003). So, with the exception of these very high Eddington ratio objects (say, with $L_{\text {bol }} / L_{\text {Edd }} \geq 0.5$ ), of which we have none in our Population 1 sample, one can neglect the second term in Eq. (10).

\subsubsection{A simple parameterization}

We see that, to a zeroth approximation, in all these cases $\Delta V_{\text {obs }}$ can be represented by the expression,

$\Delta V_{\mathrm{obs}} \approx\left(a^{2}+\sin ^{2} i\right)^{1 / 2} V_{\mathrm{Kep}}$,

where the parameter $a$ can be identified with $H / R$ or with $V_{\text {turbulent }} / V_{\mathrm{Kep}}$, and it can take a null value in the case of a warped disk.

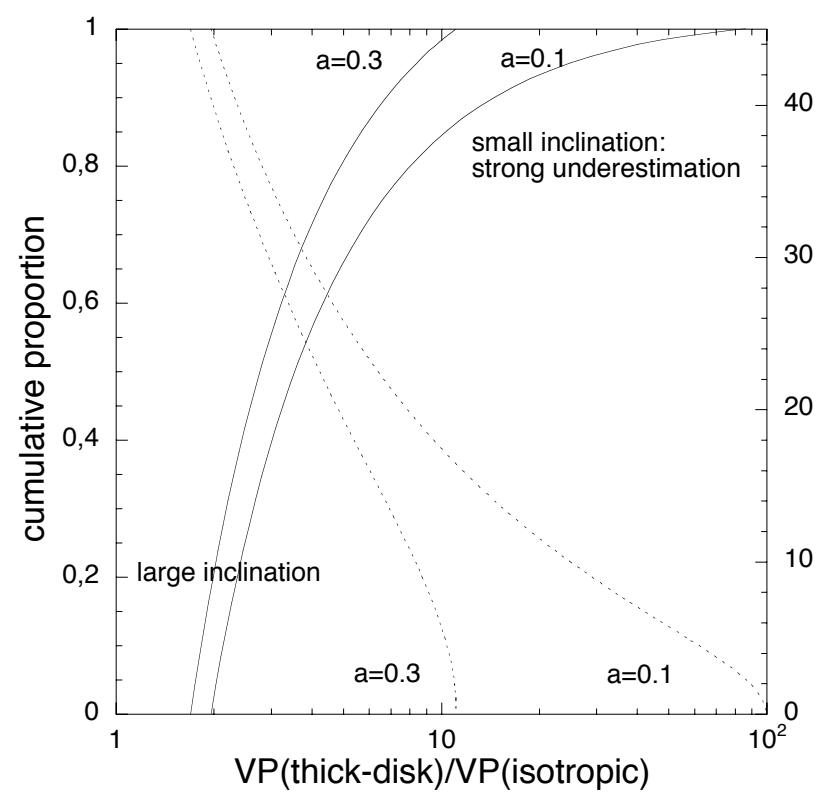

Fig. 7. The solid curves show the cumulative fraction (left axis) of AGNs that will have $A=V P_{\text {thick disk }} / V P_{\text {isotropic }}$ smaller than the value on the bottom axis for two values of the parameter $a$, assuming that the maximum inclination is $45^{\circ}$. The dotted lines show the value of $A$ for values of the inclination (right axis) over the range $0^{\circ} \leq i \leq 45^{\circ}$.

If we identify $V_{\text {Kep }}$ with the virial velocity, the virial product in this parameterization, which we will call the "generalized thick disk," or more simply "thick disk," even though we have seen that it can describe other structures, will be given by

$$
V P_{\text {thick disk }}=\frac{R_{\mathrm{BLR}} V_{\mathrm{Kep}}^{2}}{G}=\frac{R_{\mathrm{BLR}} V_{\mathrm{obs}}^{2}}{G\left(a^{2}+\sin ^{2} i\right)} .
$$

\subsection{Theoretical considerations}

The ratio of the virial product in the thick disk model $V P_{\text {thick disk }}$ to the virial product in the general case, which we will call the "isotropic case", simply because it does not have cylindrical symmetry, is equal to

$A=\frac{V P_{\text {thick disk }}}{V P_{\text {isotropic }}}=\frac{1}{a^{2}+\sin ^{2} i}$.

The value of the inclination is given as a function of $A$ in Fig. 7. We see that if the thick disk model is correct, the virial product computed in the general case could be strongly underestimated for small inclinations. The general relation can even lead to an underestimate of the mass by a factor of 100 for $a=0.1$. Note that we have neglected any correction due to possible anisotropy of the optical flux (for instance, the flux emerging from the disk can be subject to "limb-darkening" effects), or to any obscuration of the BLR. These effects should be small compared to those we are looking for.

We can compute how many objects would have a given value of $A$ by assuming that they are distributed at random for inclinations $i \leq i_{0}$. According to the unified scheme (Antonucci \& Miller 1985), Seyfert 1 nuclei are not seen edge on, and one can quite reasonably assume $i_{0} \approx 45^{\circ}$, though we have checked that our conclusions are not qualitatively changed for $i_{0}=60^{\circ}$. The probability of seeing an object at an inclination angle $i$ per unit 

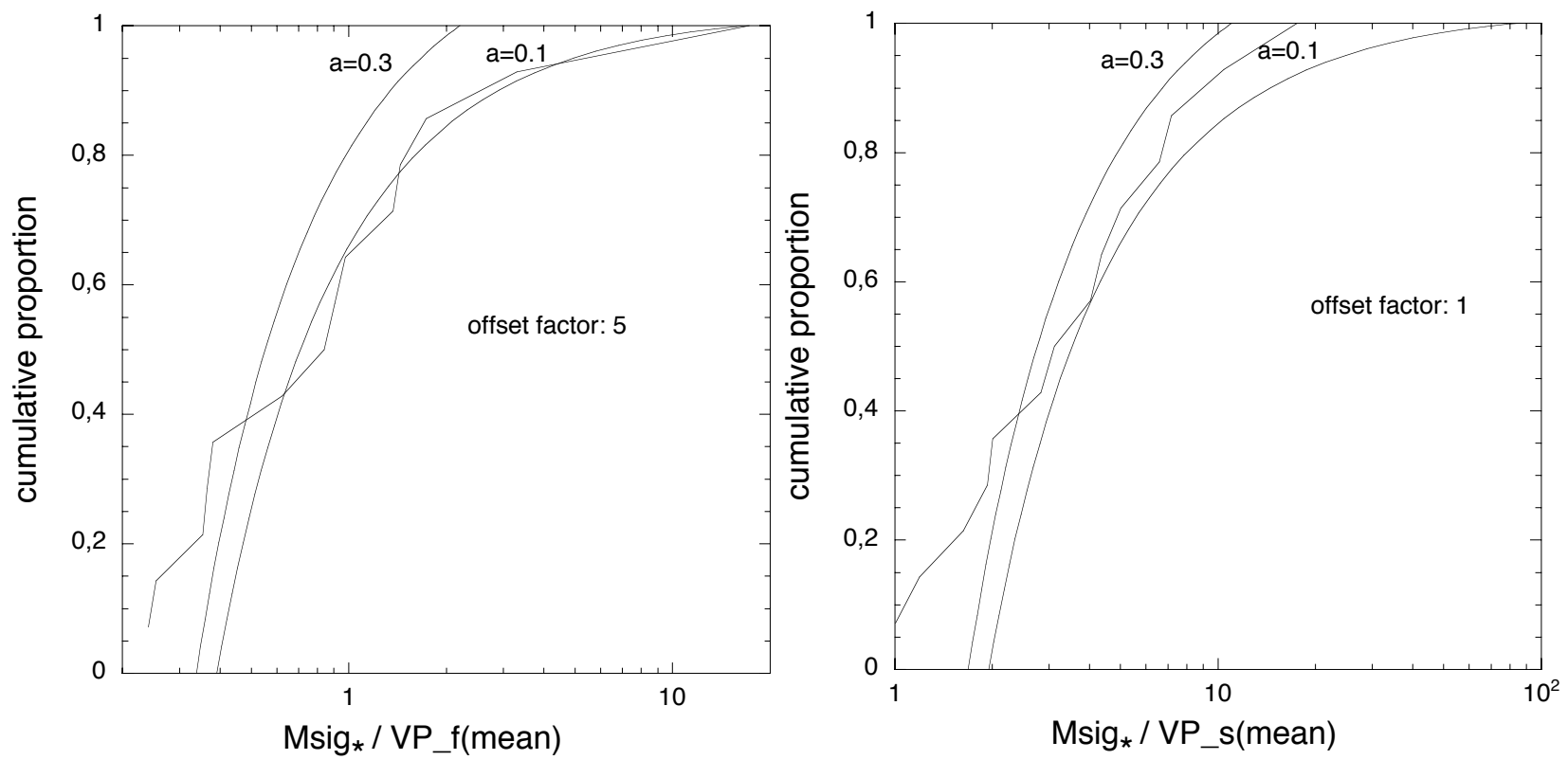

Fig. 8. Cumulative fraction of values of $M_{\sigma^{*}} / V P$ for the Onken sample of 14 objects, compared to the theoretical distribution for two thick-disk models, as shown in Fig. 7, for the $F W H M$-based values $M_{\sigma *} / V P_{\mathrm{f}}(l e f t)$ and the $\sigma_{\text {line }}$-based values $M_{\sigma^{*}} / V P_{\mathrm{s}}(r i g h t)$. The "offset factor" is described in the text.

angle interval is $\sin i /\left(1-\cos i_{0}\right)$. The number of objects per unit interval of $A$ is thus

$\frac{\mathrm{d} N}{\mathrm{~d} A}=\frac{1}{2\left(1-\cos i_{0}\right)} \frac{\left(a^{2}+\sin ^{2} i\right)^{2}}{\cos i}$.

Figure 7 shows the integral of this expression, i.e., the cumulative fraction of AGNs for which $V P_{\text {thick disk }} / V P_{\text {isotropic }}$ is smaller than a given value. For reasonable values, say $0.1 \lesssim a \lesssim 0.3, V P$ could underestimate the mass by as much as one order of magnitude in a few to several percent of the objects, specifically those which are seen at small inclinations. It is thus not implausible that a significant fraction of NLS1s, which constitute only $\sim 10 \%$ of the local AGN population, are actually seen almost "face-on," and that their Eddington ratio is consequently strongly overestimated. We elaborate on this below.

\subsection{Comparison with observations}

Some authors (Wu \& Han 2001; Zhang \& Wu 2002; McLure $\&$ Dunlop 2001) assume that the true BH masses in AGNs satisfy the $M_{\mathrm{BH}}-\sigma_{*}$ relationship and that the differences between the masses deduced from the stellar velocity dispersion $M_{\sigma *}$ and the reverberation masses $M_{\text {rev }}$ are due only to inclination effects. They then proceed to deduce the inclinations for individual AGNs, based on the discrepancies between $M_{\text {rev }}$ and $M_{\sigma *}$. Wu \& Han and Zhang \& Wu studied the reverberation-mapped objects and assumed that the BLR was a thin disk with no isotropic component of the velocity. McLure \& Dunlop studied a sample of 30 quasars where the BH masses were estimated using $F W H M$ and the $R-L$ relationship of Kaspi et al. (2000) and adopted an ad hoc complex (and rather implausible) disk geometry.

We do not think that it is possible to deduce individual inclinations in this way. It would be possible only if there were no intrinsic scatter either in the $M_{\mathrm{BH}}-\sigma_{*}$ relationship or in $M_{\mathrm{rev}}$; we know from the best-studied AGN, NGC 5548, that $V P_{\mathrm{s}}$ and thus $M_{\text {rev }}$ has an intrinsic scatter of about a factor of three.
Here we take a somewhat different approach with the goal of testing in a statistical fashion whether inclination effects might plausibly account for the distribution of differences between $M_{\text {rev }}$ and $M_{\sigma * *}$. We compare the observed distribution of $M_{\sigma *} / V P$ with the theoretical distribution $V P_{\text {thick disk }} / V P_{\text {isotropic }}$ of Fig. 7, since these should be identical to within a scaling constant (that converts $V P$ to $M_{\mathrm{rev}}$ at $i=0^{\circ}$ ) if inclination alone is responsible for the discrepancies between $M_{\mathrm{rev}}$ and $M_{\sigma *}$.

In Fig. 8, we show the cumulative distribution of the values $M_{\sigma *} / V P$ for the $F W H M$-based values of the virial product $V P_{\mathrm{f}}$ (left) and the $\sigma_{\text {line }}$-based values $V P_{\mathrm{s}}$ (right) for the Onken sample of 14 objects $^{6}$. The "offset factor" in this and subsequent figures is the number by which the theoretical $M_{\sigma *} / V P$ ratio has been divided to aid in comparison of the two distributions. It is related to, but is not identical to, the scale factor $f$ if the inclination is the sole cause of the discrepancies between $M_{\mathrm{rev}}$ and $M_{\sigma *}$, but is otherwise completely arbitrary. Figure 8 shows that the $\sigma_{\text {line }}$ - based values $M_{\sigma *} / V P_{\mathrm{s}}$ do not match the theoretical distribution, while on the contrary the $F W H M$-based values $V P_{\mathrm{f}}$ seem to match the theoretical distribution rather well at large values of $M_{\sigma *} / V P_{\mathrm{f}}$ for $a=0.1$. This suggests that the thick-disk BLR model probably has some merit, particularly in describing the line core (to which FWHM is more sensitive), implying that $F W H M$ has some dependence on inclination. However, the poor match of the theoretical and observed distributions based on $\sigma_{\text {line }}$ implies that $\sigma_{\text {line }}$ is insensitive to source inclination. We speculate further in Sect. 4.

We now consider separately Populations 1 and 2 . This unfortunately exacerbates the problems associated with small-number statistics, but we find some important differences between the two populations that we believe are enlightening. Figure 9 shows the cumulative distribution of $M_{\sigma *} / V P_{\mathrm{f}}$ for Population 2, although we have actually relaxed our orginal arbitrary criterion

${ }^{6}$ Note that for the sake of simplicity, we will restrict the discussion only to quantities derived from the mean spectra since these do not differ significantly from those derived from the rms spectra. Also, in the cases of sources for which multiple datasets are available, we use an average value of $V P$ so that each source is counted only once. 


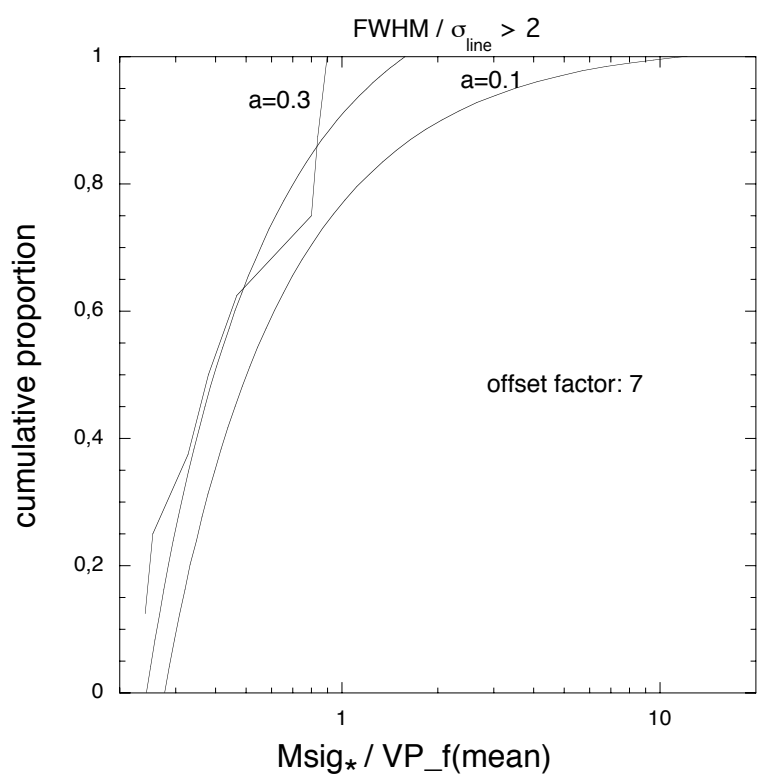

Fig. 9. Cumulative fraction of $M_{\sigma^{*}} / V P_{\mathrm{f}}$ for Population 2 objects (expanded to include all objects with $F W H M / \sigma_{\text {line }}>2$ ).

$F W H M / \sigma_{\text {line }}>2.35$ to $F W H M / \sigma_{\text {line }}>2$ in order to increase the sample size. Figure 9 shows that the cumulative distribution is not well-described by the theoretical curve at large values of $M_{\sigma *} / V P_{\mathrm{f}}$. We conclude that our generalized thick-disk model with $i<45^{\circ}$ is not a good description for this population. More specifically, the distribution of $M_{\sigma *} / V P_{\mathrm{f}}$ does not appear to be controlled by inclination angle for this population.

In contrast, the cumulative distribution of Population 1 shown in Fig. 10 is well-described by the theoretical curve at large values of $M_{\sigma *} / V P_{\mathrm{f}}$ for the case $a=0.1$. This figure shows clearly that the few AGNs with $F W H M / \sigma_{\text {line }}<2$ seem to fit the theoretical distribution very well, but the AGNs with $2<F W H M / \sigma_{\text {line }}<2.35$ match the theoretical distribution rather less well.

We conclude that among the sample of AGNs with small $F W H M / \sigma_{\text {line }}$ ratios, the three objects with the highest values of $M_{\sigma *} / V P_{\mathrm{f}}$ (NGC 4051, Mrk 590 and NGC 7469) are probably actually observed at low inclination. Although the statistics are very poor, we are led to the conclusion that the difference in scale factor $f$ (Table 2) between Population 1/A and Population 2/B is due at least partly to an inclination effect. According to Fig. 7, and taking into account the offset factor, these three objects are probably at inclinations $i \lesssim 20^{\circ}$, and their masses $M_{\mathrm{rev}}$ could be underestimated by factors as large as an order of magnitude.

While some properties of NLS1s can be attributed to either high Eddington ratios or low inclination, a clear consensus based on the preponderance of evidence is that accretion rate is probably the key factor that defines the NLS1 class (see Boller 2000). While we agree that some properties of NLS1s, in particular their rapid and large amplitude X-ray variability, steep X-ray spectra, and the purported larger fraction of bars in their host galaxies (Crenshaw et al. 2003; Ohta et al., in preparation) can certainly not be explained only by the inclination, we argue that inclination does play an important, although not the dominant, role in defining this class of object. In this context, we point out that Williams et al. (2004) show that not all optically selected NLS1s seem to be high accretion sources; many have characteristics more typical of broad-line objects. These may well be the sources for which inclination is important.

\subsection{Inclinations of individual objects}

Unfortunately, there is currently no way to measure the inclination of any AGN BLR to any reasonable accuracy, but there are few observables that are even good indicators of inclination. Of the seven Population 2 objects in the Onken sample, estimates are available for only two AGNs, 3C 390.3 and NGC 4151. The former is one of the relatively rare strongly double-peaked emitters; the double peaks are widely regarded as the signature of an inclined disk in Keplerian rotation. Eracleous et al. (1996), based on several indicators, conclude that the inclination of the BLR in this system must be in the range $19<i<42^{\circ}$, and based on the profile fitting alone, Eracleous \& Halpern (1994) find that $i=24-30^{\circ}$. At the very least, this relatively large inclination is consistent with our finding that 3C 390.3 is not a member of Population 1. On the other hand, it is quite natural to find a relatively large inclination for a powerful FRII radio source like 3C 390.3 that does not show superluminal motion. In the case of NGC 4151, the deduced inclination ranges from $12-21^{\circ}$ (Boksenberg et al. 1995) and 18-23 (Winge et al. 1999), to as high as $40^{\circ}$ (Pedlar et al. 1998) or even $70^{\circ}$ (Kaiser et al. 2000). There is thus no clear consensus on the inclination of this system.

There are also few inclination estimates available for the Population 1 objects. The radio source 3C 120 has a superluminal jet, and therefore must be seen fairly close to face-on. Marscher et al. (2002) estimate that the inclination of this source must be less than $20^{\circ}$, which is consistent with the fact that it is a Population 1 object and with Fig. 10. In the case of Mrk 110, Kollatschny (2003b) showed that the variable part of the broad lines of this bright NLS1 nucleus are redshifted with respect to the systemic velocity, from which he deduced a "gravitational mass" of $1.4 \times 10^{8} M_{\odot}$, larger than the "isotropic" reverberation mass by almost one order of magnitude, and requiring an inclination angle $i \approx 19^{\circ}$. The position of Mrk 110 in Fig. 10 indicates a relatively large inclination angle, but we have seen that the objects located in this part of the diagram do not appear to be very sensitive to inclination, and are therefore subject to large uncertainties in the determination of the inclination.

Popović et al. (2004) attempted to determine inclinations for individual objects by detailed fitting of individual line profiles. Unfortunately, their model has more free parameters than observational constraints and these authors are obliged to impose at least one arbitrary constraint, which they choose to be the emissivity as a function of the radius. They generally find quite small inclinations, but with large uncertainties, and inescapable model dependence.

\subsection{The influence of inclination on the scale factor}

The similarity between the offset factor of 3 found for the Population1/A objects in Fig. 10 and the mass scale factor of 2 to 2.5 of this population (Table 2) could give the impression that the scale factor is determined mainly by inclination, but this is not the case. If indeed the inclination effect were the principal factor affecting the scale factor $f$, then the average ratio would be

$$
\left\langle\frac{V P_{\text {thick disk }}}{V P_{\text {isotropic }}}\right\rangle=\frac{\int A(i) \sin i \mathrm{~d} i}{1-\cos i_{0}}
$$

Using Eq. (13), we obtain

$$
\begin{aligned}
\left\langle\frac{V P_{\text {thick disk }}}{V P_{\text {isotropic }}}\right\rangle= & \frac{1}{2 b\left(1-\cos i_{0}\right)} \\
& \times\left[\ln \left(\frac{b+1}{b+\cos i_{0}}\right)+\ln \left(\frac{b-\cos i_{0}}{b-1}\right)\right],
\end{aligned}
$$




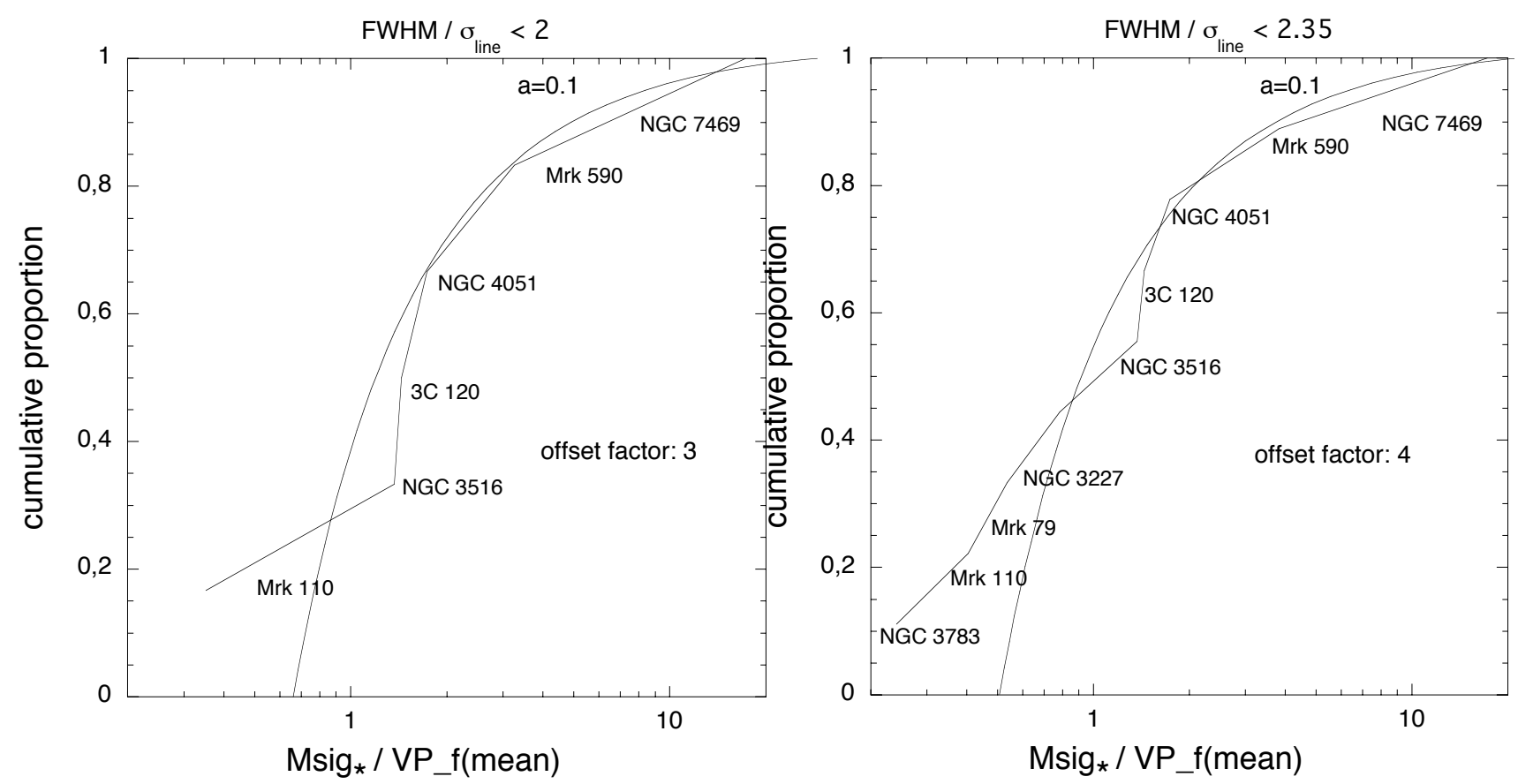

Fig. 10. Cumulative fraction $M_{\sigma^{*}} / V P_{\mathrm{f}}$ for Population 1 AGNs in the Onken sample, compared to the predicted distribution for our generalized thick-disk model with $a=0.1$. In the left panel, Population 1 is restricted to AGNs with $F W H M / \sigma_{\text {line }}<2$, while in the right panel, AGNs with $2<F W H M / \sigma_{\text {line }}<2.35$ are also included.

where $b=\left(1+\langle a\rangle^{2}\right)^{1 / 2}$ and $\langle a\rangle$ is the inclination averaged value of $a$. For $\langle a\rangle=0.1$ and $i_{0}=45^{\circ}$, we find $\left\langle V P_{\text {thick disk }} / V P_{\text {isotropic }}\right\rangle=7.1$ (or 4.8 for $a=0.2$ ). This is larger than the scale factor for Population 1/A, which means that while the inclination does certainly play a role in the determining the scale factor $f$, it is not the dominant source of the differences between $M_{\text {rev }}$ and $M_{\sigma *}$.

\section{Discussion}

An obvious question to ask is why the $F W H M$ would be more dependent on the inclination than the line dispersion $\sigma_{\text {line }}$ ? While a definitive answer is not possible, we speculate that the line wings, to which $\sigma_{\text {line }}$ is relatively more sensitive, arise primarily in a more-or-less isotropic component, perhaps in the form of a "disk wind". The line core, to which $F W H M$ is more sensitive, might then arise primarily in a Keplerian disk component, and thus $F W H M$ would be more sensitive to inclination. Such a scenario would also account, at least in part, for the smaller values of $F W H M / \sigma_{\text {line }}$ ratios in Population 1 objects, as the presence of stronger winds in Population 1 would naturally correspond to higher Eddington ratios than Population 2. By analogy, we know indeed that hot stars radiating close to their Eddington limit have strong winds, and there seems to be an emerging consensus that this is also the case for quasars accreting at a high Eddington ratio (King \& Pounds 2003; Pounds et al. 2003).

We can further speculate as to how the BLR can differ between low and high Eddington ratio objects. At large distances from the center, the disk is self-gravitating and gravitationally unstable (see Collin \& Huré 2001). As a consequence of the gravitational instability, the disk should be broken into clumps. The fate of these clumps, and more generally the state of the disk in this region, the accretion mechanism, and the way angular momentum is removed, are unknown (e.g., Collin \& Kawaguchi 2004), but we might speculate that the "disk" would be made of discrete clumps and it seems natural to identify these clumps

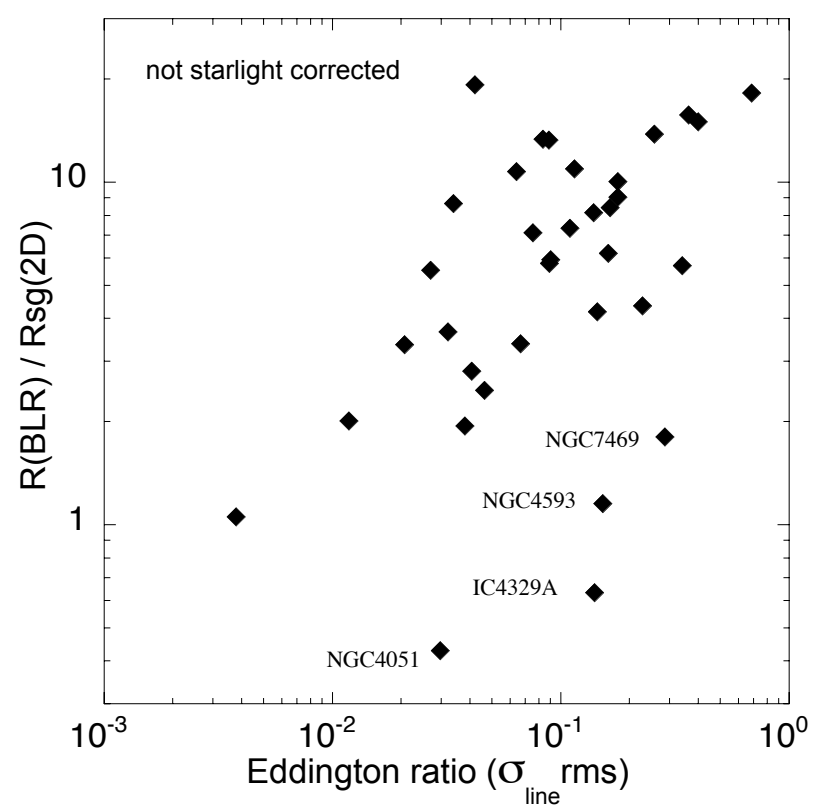

Fig. 11. The ratio $R_{\mathrm{BLR}} / R_{\mathrm{sg}}$, as described in the text. The four outliers are objects whose luminosity and Eddington ratio are probably overestimated (see the text). If $R_{\mathrm{BLR}} / R_{\mathrm{sg}}>4$, the BLR is gravitationally unstable.

with the BLR clouds. The heating of the cloud system would be thus provided by the collisions between the clouds, as suggested also for the molecular torus (Krolik \& Begelman 1988). The larger the gravitational instability, the larger the heating rate (cf. Lodato \& Rice 2004). It is also probable that a fraction of the clumps constitute the basis of a wind, which would be more efficient when the gravitational instability is strong.

Figure 11 displays the ratios $R_{\mathrm{BLR}} / R_{\mathrm{sg}}$ versus the Eddington ratio for the reverberation-mapped sample, where $R_{\mathrm{sg}}$ 
is the radius above which the self-gravity of the disk overcomes the vertical component of the central gravity. Here $R_{\mathrm{sg}}$ has been computed with a $2 \mathrm{D}$ simulation using real opacities (Huré 1998) ${ }^{7}$ and assuming a viscosity coefficient $\alpha=$ 0.1 . There is a clear correlation between $R_{\mathrm{BLR}} / R_{\mathrm{sg}}$ and the Eddington ratio, with the exception of the four outliers labeled in the figure; three are among the least-luminous AGNs in the sample, so their luminosity could have been overestimated as mentioned in Sect. 2, and NGC 7469 is the object suspected to have the largest inclination effect, so its mass could be strongly underestimated, and thus its Eddington ratio be also overestimated. The radius at which the disk becomes gravitationnally unstable is about four times larger than $R_{\mathrm{sg}}$. Thus, according to Fig. 11, the BLR lies in the gravitationnally unstable region in the objects with $L / L_{\text {Edd }} \gtrsim 0.1$. On the other hand, objects with low Eddington ratios $\left(L / L_{\text {Edd }} \lesssim 0.03\right)$ have their BLRs in the gravitationnally stable region. We therefore speculate that the BLR of Population 1/A AGNs, which is gravitationally unstable, is more influenced by the wind than Population 2/B, thus explaining the difference in the line profiles and the difference in the $F W H M / \sigma_{\text {line }}$ ratio.

Another possible explanation for why the $F W H M / \sigma_{\text {line }}$ ratio is larger in Population 2/B objects is that the structure of the inner accretion disk is different in these objects. Population 2/B includes objects like the broad-line radio galaxy 3C 390.3, which have strongly double-peaked line profiles. Chen \& Halpern (1989) have suggested that the inner disk is an inflated ionsupported torus which illuminates the outer line-emitting part of the extended disk. It is indeed thought that a hot advection dominated accretion flow (ADAF, see Narayan \& Yi 1994 and subsequent works), or more generally a radiatively inefficient accretion flow (RIAF), is present close to the $\mathrm{BH}$, and that such structures become increasingly prominent with decreasing Eddington ratio. If the Eddington ratio is small, we expect that an annular region at relatively small distance from the $\mathrm{BH}$ is heated by the X-rays from the geometrically thick RIAF, giving rise to very broad two-peaked line profiles. Note however that in this case, the line-emitting region would be a thin disk, which is very sensitive to the inclination, contrary to what we have deduced previously.

Finally, Murray \& Chiang (1997) argue that a varying optical depth in outflowing disk winds can explain the presence of single or double peaked line profiles. In this scenario, a low optical depth would tend to generate double-peaked lines while singlepeaked profiles are the result of high optical depths in the wind.

Could a combination of these phenomena (gravitational instability, inflated inner hot disk, wind) which are all linked with the Eddington ratio, combined with the influence of inclination, explain the variation of the $F W H M / \sigma_{\text {line }}$ ratio among the AGN population? Obviously it will be necessary to consider larger samples of objects for which these two parameters are available in order to check these different ideas.

\section{Summary}

In this contribution, we have initiated a study of the relationship between AGN BH masses and other physical properties of AGNs that can be discerned from broad emission-line profiles. We have used the ratio of $F W H M$ to the line dispersion $\sigma_{\text {line }}$ to characterize the emission-line profiles and have shown that this

\footnotetext{
7 In the course of these computations, we have confirmed that the analytical formulae for $R_{\mathrm{sg}}$ given by Kawaguchi et al. (2004a) show consistent results (with systematically larger values by a factor of $\sim 1.5$ ).
}

ratio is anticorrelated with Eddington ratio and with line widths; broader emission lines tend to have relatively flat-topped profiles, and narrower lines have more extended wings. We separate AGNs into two populations on the basis of their $\mathrm{H} \beta$ profiles, a Population 1 with $F W H M / \sigma_{\text {line }}<2.35$ and a Population 2 with $F W H M / \sigma_{\text {line }}>2.35$. Not surprisingly, these two populations overlap strongly with Populations A and B of Sulentic et al. (2000) which are separated by $F W H M$ only.

We then make the assumption that AGNs follow the same $M_{\mathrm{BH}}-\sigma_{*}$ relationship as quiescent galaxies and scale the virial product, the observable parameter, to determine the statistical value of the scaling factor $\langle f\rangle$ of Eq. (1) (cf. Onken et al. 2004). We do this for virial products based on both $\sigma_{\text {line }}$ and $F W H M$, as measured in both the mean and rms spectra. We find that, to within the uncertainties, the scaling factor is constant for both Populations 1 and 2 for virial products based on using $\sigma_{\text {line }}$ as the line-width measure. On the other hand, the scaling factors are significantly different for the two populations if the virial product is based on FWHM. This means that $\sigma_{\text {line }}$ is a less biased mass estimator than is FWHM. However, we show that it is possible to remove heuristically the bias in masses based on FWHM and obtain masses estimates consistent with those based on $\sigma_{\text {line }}$.

For the 14 objects with measured bulge velocity dispersions $\sigma_{*}$, we have compared the black hole mass predicted by the $M_{\mathrm{BH}}-\sigma_{*}$ relationship with the mass determined by reverberation mapping. By comparing the distribution of the ratio of these masses with the distribution expected from a generalized thick disk model of the BLR, we find statistical evidence that in some small fraction of cases, the reverberation-based BH masses in Population 1 objects are underestimated on account of inclination effects. We find no evidence for inclination effects in Population 2 objects. We speculate that the difference between the two populations is the relative strength of a disk-wind component, which is stronger in Population 1. Finally, we discuss the possible role of self-gravity as the physical driver controlling the strength of the disk wind. We find a stronger wind is expected for larger Eddington ratios, which is consistent with the smaller $F W H M / \sigma_{\text {line }}$ ratios found for Population 1 objects.

Acknowledgements. We would like to thank Jean-Marc Huré and Didier Pelat for useful discussions. T.K. is grateful for financial support through a JSPS Postdoctoral Fellowship. This research was supported in part by the US National Science Foundation through grant AST-0205964 (B.M.P.) and AST-0307384 (M.V.) and by NASA through grant HST-AR-10691 from the Space Telescope Science Institute (M.V. and B.M.P.).

\section{References}

Antonucci, R. R. J., \& Miller, J. S. 1985, ApJ, 297, 621

Aoki, K., Kawaguchi, T., \& Ohta, K. 2005, ApJ, 618, 601

Barth, A. J., Greene, J. E., \& Ho, L. C. 2005, ApJ, 619, L151

Bentz, M. C., Peterson, B. M., Pogge, R. W., Vestergaard, M., \& Onken, C. A. 2006, ApJ, in press

Blandford, R. D., \& McKee, C. F. 1982, ApJ, 255, 419

Boksenberg, A., Catchpole, R. M., Macchetto, F., et al. 1995, ApJ, 440, 151

Boller, T. 2000, New Astronomy Review, 44

Boroson, T. A. 2002, ApJ, 565, 78

Boroson, T. A. 2003, ApJ, 585, 647

Boroson, T. A., \& Green, R. F. 1992, ApJS, 80, 109

Chen, K., \& Halpern, J. P. 1989, ApJ, 344, 115

Chevallier, L., Collin, S., Dumont, A.-M., et al. 2005, A\&A, in press

Clavel, J. 1991, in Variability of Active Galaxies, ed. W. J. Duschl, S. J. Wagner,

\& M. Camenzind (Berlin: Springer-Verlag), 31

Collin, S., \& Huré, J.-M. 2001, A\&A, 372, 50

Collin, S., \& Kawaguchi, T. 2004, A\&A, 426, 797

Collin, S., Boisson, C., Mouchet, M., et al. 2002, A\&A, 388, 771 (C02)

Collin-Souffrin, S., \& Lasota, J. 1988, PASP, 100, 1041

Crenshaw, D. M., Kraemer, S. B., \& Gabel, J. R. 2003, AJ, 126, 1690

Dumont, A. M., \& Collin-Souffrin, S. 1990, A\&A, 229, 313 
Elvis, M. 2000, ApJ, 545, 63

Elvis, M., Wilkes, B. J., McDowell, J. C., et al. 1994, ApJS, 95, 1

Eracleous, M., \& Halpern, J. P. 1994, ApJS, 90, 1

Eracleous, M., Halpern, J. P., \& Livio, M. 1996, ApJ, 459, 89

Ferrarese, L., \& Merritt, D. 2000, ApJ, 539, L9

Ferrarese, L., Pogge, R. W., Peterson, B. M., et al. 2001, ApJ, 555, L79

Fromerth, M. J., \& Melia, F. 2000, ApJ, 533, 172

Gebhardt, K., Bender, R., Bower, G., et al. 2000a, ApJ, 539, L13

Gebhardt, K., Kormendy, J., Ho, L. C., et al. 2000b, ApJ, 543, L5

Gierliński, M., \& Done, C. 2004, MNRAS, 349, L7

Greene, J. E., \& Ho, L. C. 2004, ApJ, 610, 722

Greenhill, L. J., Henkel, C., Becker, R., Wilson, T. L., \& Wouterloot, J. G. A. 1995, A\&A, 304, 21

Horne, K., Peterson, B. M., Collier, S. J., \& Netzer, H. 2004, PASP, 116, 465

Huré, J. 1998, A\&A, 337, 625

Jackson, N., \& Browne, I. W. A. 1991, MNRAS, 250, 422

Kaiser, M. E., Bradley, L. D., II, Hutchings, J. B., et al. 2000, ApJ, 528, 260

Kaspi, S., Smith, P. S., Netzer, H., et al. 2000, ApJ, 533, 631

Kaspi, S., Maoz, D., Netzer, H., et al. 2005, ApJ, 629, 61

Kawaguchi, T. 2003, ApJ, 593, 69

Kawaguchi, T., Pierens, A., \& Huré, J.-M. 2004a, A\&A, 415, 47

Kawaguchi, T., Aoki, K., Ohta, K., \& Collin, S. 2004b, A\&A, 420, L23

King, A. R., \& Pounds, K. A. 2003, MNRAS, 345, 657

Kollatschny, W. 2003a, A\&A, 407, 461

Kollatschny, W. 2003b, A\&A, 412, L61

Kollmeier, J. A., Onken, C. A., Kochanek, C. S., et al. 2006, ApJ, submitted [arXiv: astro-ph/0508657]

Krolik, J. H. 2001, ApJ, 551, 72

Krolik, J. H., \& Begelman, M. C. 1988, ApJ, 329, 702

Lodato, G., \& Rice, W. K. M. 2004, MNRAS, 351, 630

Marscher, A. P., Jorstad, S. G., Gómez, J.-L., et al. 2002, Nature, 417, 625

Mathur, S., Kuraszkiewicz, J., \& Czerny, B. 2001, New Astron., 6, 321

McLure, R. J., \& Dunlop, J. S. 2001, MNRAS, 327, 199

McLure, R. J., \& Jarvis, M. J. 2002, MNRAS, 337, 109

Merritt, D., \& Ferrarese, L. 2001, ApJ, 547, 140

Miyoshi, M., Moran, J., Herrnstein, J., et al. 1995, Nature, 373, 127

Murray, N., \& Chiang, J. 1995, ApJ, 454, L105

Murray, N., \& Chiang, J. 1997, ApJ, 474, 91

Murray, N., \& Chiang, J. 1998, ApJ, 494, 125

Narayan, R., \& Yi, I. 1994, ApJ, 428, L13

Nelson, C. H., Green, R. F., Bower, G., Gebhardt, K., \& Weistrop, D. 2004, ApJ, 615,652

Netzer, H. 1990, in Active Galactic Nuclei, ed. T. J.-L. Courvoisier, \& M. Mayor (Berlin: Springer Verlag), 137
Netzer, H. 2003, ApJ, 583, L5

Netzer, H., Maoz, D., Laor, A., et al. 1990, ApJ, 353, 108

Onken, C. A., Ferrarese, L., Merritt, D., et al. 2004, ApJ, 615, 645

Onken, C. A., \& Peterson, B. M. 2002, ApJ, 572, 746

Pedlar, A., Fernandez, B., Hamilton, N. G., Redman, M. P., \& Dewdney, P. E. 1998, MNRAS, 300, 1071

Peterson, B. M. 1993, PASP, 105, 247

Peterson, B. M., \& Wandel, A. 1999, ApJ, 521, L95

Peterson, B. M., \& Wandel, A. 2000, ApJ, 540, L13

Peterson, B. M., Berlind, P., Bertram, R., et al. 2002, ApJ, 581, 197

Peterson, B. M., Ferrarese, L., Gilbert, K. M., et al. 2004, ApJ, 613, 682

Popović, L. Č., Mediavilla, E., Bon, E., \& Ilić, D. 2004, A\&A, 423, 909

Pounds, K. A., Reeves, J. N., King, A. R., et al. 2003, MNRAS, 345, 705

Proga, D., \& Kallman, T. R. 2004, ApJ, 616, 688

Reynolds, C. S., \& Wilms, J. 2000, ApJ, 533, 821

Rokaki, E., Lawrence, A., Economou, F., \& Mastichiadis, A. 2003, MNRAS, 340, 1298

Sergeev, S. G., Pronik, V. I., Sergeeva, E. A., \& Malkov, Yu. F. 1999, AJ, 118, 2658

Shakura, N. I., \& Sunyaev, R. A. 1973, A\&A, 24, 337

Shapovalova, A. I., Doroshenko, V. T., Bochkarev, N. G., et al. 2004, A\&A, 422, 925

Shields, J. C., Ferland, G. J., \& Peterson, B. M. 1995, ApJ, 441, 507

Smith, J. E., Robinson, A., Young, S., Axon, D. J., \& Corbett, E. A. 2005 [arXiv:astro-ph/0502080]

Sulentic, J. W., Zwitter, T., Marziani, P., \& Dultzin-Hacyan, D. 2000, ApJ, 536, L5

Tremaine, S., Gebhardt, K., Bender, R., et al. 2002, ApJ, 574, 740

Vestergaard, M. 2002, ApJ, 571, 733

Vestergaard, M. 2004, ApJ, 601, 676

Vestergaard, M., \& Peterson, B. M. 2005, ApJ, 625, 688

Vestergaard, M., \& Peterson, B. M. 2006, ApJ, in press

Vestergaard, M., Wilkes, B. J., \& Barthel, P. D. 2000, ApJ, 538, L103

Wandel, A., Peterson, B. M., \& Malkan, M. A. 1999, ApJ, 526, 579

Wandel, A. 2001, Probing the Physics of Active Galactic Nuclei, ASP Conf. Ser., 224,365

Wijers, R. A. M. J., \& Pringle, J. E. 1999, MNRAS, 308, 207

Williams, R. J., Mathur, S., \& Pogge, R. W. 2004, ApJ, 610, 737

Wills, B. J., \& Browne, I. W. A. 1986, ApJ, 302, 56

Winge, C., Axon, D. J., Macchetto, F. D., et al. 1999, ApJ, 519, 134

Wu, X., \& Han, J. L. 2001, ApJ, 561, L59

Yu, Q., \& Tremaine, S. 2002, MNRAS, 335, 965

Zamanov, R., Marziani, P., Sulentic, J. W., et al., 2002, ApJ, 576, L9

Zhang, T.-Z., \& Wu, X.-B. 2002, Chin. J. Astron. Astrophys., 2, 487 